\title{
The proof of the Nirenberg-Treves conjecture
}

\author{
Nils Dencker
}

\begin{abstract}
We prove the Nirenberg-Treves conjecture: that for principal type pseudodifferential operators local solvability is equivalent to condition $(\Psi)$. This condition rules out certain sign changes of the imaginary part of the principal symbol along the bicharacteristics of the real part. We obtain local solvability by proving a localizable estimate for the adjoint operator with a loss of two derivatives (compared with the elliptic case).

The proof involves a new metric in the Weyl (or Beals-Fefferman) calculus. This makes it possible to reduce to the case when the gradient of the imaginary part is non-vanishing, and then the zeroes form a smooth submanifold. The estimate uses a new type of weight, which measures the change of the distance to the zeroes of the imaginary part along the bicharacteristics of the real part between the minima of the curvature of this submanifold. By using condition $(\Psi)$ and this weight, we can construct a multiplier which gives the estimate.
\end{abstract}

\section{Introduction}

We shall study the question of local solvability of a classical pseudo-differential operator $P \in \Psi_{c l}^{m}(M)$ on a $C^{\infty}$ manifold $M$. Thus, we assume that the symbol of $P$ is an asymptotic sum of homogeneous terms, and that $p=\sigma(P)$ is the homogeneous principal symbol of $P$. We shall also assume that $P$ is of principal type, which means that the Hamilton vector field $H_{p}$ and the radial vector field are linearly independent when $p=0$.

Local solvability of $P$ at a compact set $K \subseteq M$ means that the equation

$$
P u=v
$$

has a local solution $u \in \mathcal{D}^{\prime}(M)$ in a neighborhood of $K$ for any $v \in C^{\infty}(M)$ in a set of finite codimension. We can also define microlocal solvability at any compactly based cone $K \subset T^{*} M$, see Definition 26.4.3 in [10].

MSC 2000 : 35S05 (primary) 35A07, 58J40, 47G30 (secondary). 
It was conjectured by Nirenberg and Treves [19] that condition $(\Psi)$ was equivalent to local solvability of pseudo-differential operators of principal type. Condition $(\Psi)$ means that

$\operatorname{Im}(a p)$ does not change sign from - to + along the oriented bicharacteristics of $\operatorname{Re}(a p)$

for any $0 \neq a \in C^{\infty}\left(T^{*} M\right)$; actually it suffices to check this for some $a \in C^{\infty}\left(T^{*} M\right)$ such that $H_{\operatorname{Re}(a p)} \neq 0$ by Theorem 26.4.12 in [10]. By oriented bicharacteristics of $\operatorname{Re}(a p)$ we mean the positive flow-out of the Hamilton vector field $H_{\operatorname{Re}(a p)} \neq 0$ on $\operatorname{Re}(a p)=0$, these are also called semi-bicharacteristics. Condition (1.2) is invariant under conjugation with elliptic Fourier integral operators and multiplication with elliptic pseudo-differential operators, see Lemma 26.4.10 in [10].

For differential operators, condition $(\Psi)$ is equivalent to condition $(P)$, which rules out any sign changes of $\operatorname{Im}(a p)$ along the bicharacteristics of $\operatorname{Re}(a p)$ for $0 \neq$ $a \in C^{\infty}\left(T^{*} M\right)$. The sufficiency of $(P)$ for local solvability of principal type pseudodifferential operators was proved by Nirenberg and Treves [19] in the case when the principal symbol is real analytic, and by Beals and Fefferman [1] in the general case.

The necessity of $(\Psi)$ for local solvability of principal type pseudo-differential operators was proved by Moyer in two dimensions and by Hörmander in general, see Corollary 26.4.8 in [10]. In the analytic category, the sufficiency of condition $(\Psi)$ for solvability of principal type microdifferential operators acting on microfunctions was proved by Trépreau [20] (see also [11, Chapter VII]). The sufficiency of condition ( $\Psi$ ) for local solvability of principal type pseudo-differential operators in two dimensions was proved by Lerner [13], leaving the higher dimensional case open.

Lerner [14] constructed counterexamples to the sufficiency of $(\Psi)$ for local optimal $\left(L^{2}\right)$ solvability of first order principal type pseudo-differential operators, raising doubts on whether the condition really was sufficient for solvability. But it was proved by the author [4] that Lerner's counterexamples are locally solvable with loss of at most two derivatives (compared with the elliptic case). Observe that optimal solvability of first order principal type pseudo-differential operators means a loss of one derivative. There are several results giving local solvability under conditions stronger than $(\Psi)$, see [5], [12], [15] and [17].

In this paper we shall prove local solvability of principal type pseudo-differential operators $P \in \Psi_{c l}^{m}(M)$ satisfying condition $(\Psi)$, this resolves the Nirenberg-Treves conjecture. To get local solvability we shall assume a strong form of the non-trapping condition at $x_{0}$ : that all semi-characteristics are transversal to the fiber $T_{x_{0}}^{*} \mathbf{R}^{n}$, i.e., $p\left(x_{0}, \xi\right)=0 \Longrightarrow \partial_{\xi} p\left(x_{0}, \xi\right) \neq 0$.

Theorem 1.1. If $P \in \Psi_{c l}^{m}(M)$ is of principal type satisfying condition ( $\left.\Psi\right)$ near $x_{0} \in M$ and $\partial_{\xi} p\left(x_{0}, \xi\right) \neq 0$ when $p\left(x_{0}, \xi\right)=0$, then $P$ is locally solvable at $x_{0}$.

It follows from the proof that we lose at most two derivatives in the estimate of the adjoint, which is one more compared with the condition $(P)$ case. Thus the result has the consequence that hypoelliptic operators of principal type can lose at most two derivatives. In fact, if the operator is hypoelliptic of principal type, then 
the adjoint is solvable of principal type, thus satisfying condition $(\Psi)$ and we obtain an estimate of the operator.

Theorem 1.1 is going to be proved by the construction of a pseudo-sign which will be used in a multiplier estimate. The symbol of the pseudo-sign is, modulo elliptic factors, essentially a perturbation of the signed homogeneous distance to the sign changes of the imaginary part of the principal symbol.

Observe that Theorem 1.1 can be microlocalized: if condition $(\Psi)$ holds microlocally near $\left(x_{0}, \xi_{0}\right) \in S^{*}(M)$ then $P$ is microlocally solvable near $\left(x_{0}, \xi_{0}\right)$, see Corollary 2.4. Since we lose two derivatives in the estimate this is not trivial, it is a consequence of the special type of estimate (see Remark 2.3). This paper is a shortened version of [7], we have excluded some of the longer and more technical proofs.

We would like to thank Lars Hörmander and Nicolas Lerner for valuable comments leading to corrections and improvements of the proof.

\section{Reduction to the multiplier estimate}

In this section we shall reduce the proof of Theorem 1.1 to an estimate for a microlocal normal form of the adjoint for the operator. By using Darboux' theorem and the Malgrange Preparation Theorem, we may obtain the adjoint $P^{*}$ on the following microlocal normal form

$$
P_{0}=D_{t}+i F\left(t, x, D_{x}\right)
$$

where $F \in C\left(\mathbf{R}, \Psi_{c l}^{1}\left(T^{*} \mathbf{R}^{n}\right)\right)$ has real principal symbol $\sigma(F)=f$. Observe that we do not assume that $t \mapsto f(t, x, \xi)$ is differentiable. Since $P$ satisfies condition $(\Psi)$ we find that $P_{0}$ satisfies condition $(\bar{\Psi})$ :

$$
t \mapsto f(t, x, \xi) \text { does not change sign from }+ \text { to - with increasing } t \text { for any }(x, \xi) \text {. }
$$

We shall use the Weyl quantization of symbols $a(x, \xi) \in C^{\infty}\left(T^{*} \mathbf{R}^{n}\right) \bigcap \mathcal{S}^{\prime}\left(T^{*} \mathbf{R}^{n}\right)$ :

$$
a^{w}\left(x, D_{x}\right) u(x)=(2 \pi)^{-n} \iint \exp (i\langle x-y, \xi\rangle) a\left(\frac{x+y}{2}, \xi\right) u(y) d y d \xi \quad u \in C_{0}^{\infty}\left(\mathbf{R}^{n}\right) .
$$

For Weyl calculus notations and results, see [10, Section 18.5]. Observe that $\operatorname{Re} a^{w}=$ $(\operatorname{Re} a)^{w}$ is the symmetric part and $i \operatorname{Im} a^{w}=(i \operatorname{Im} a)^{w}$ the antisymmetric part of the operator $a^{w}$. Also, if $a \in S_{1,0}^{m}\left(T^{*} \mathbf{R}^{n}\right)$ then $a\left(x, D_{x}\right) \cong a^{w}\left(x, D_{x}\right)$ modulo $\Psi_{1,0}^{m-1}\left(T^{*} \mathbf{R}^{n}\right)$. In the following, we shall denote $S_{\varrho, \delta}^{m}\left(T^{*} \mathbf{R}^{n}\right)$ by $S_{\varrho, \delta}^{m}, 0 \leq \delta \leq \varrho \leq 1$.

Definition 2.1. We say that the symbol $b(x, \xi)$ is in $S_{1 / 2,1 / 2}^{m}$ of first order, if $b$ satisfies the estimates in $S_{1 / 2,1 / 2}^{m}$ for derivatives of order $\geq 1$.

This means that the homogeneous gradient $\left(\partial_{x} b,|\xi| \partial_{\xi} b\right) \in S_{1 / 2,1 / 2}^{m+\frac{1}{2}}$, and implies that the commutators of $b^{w}$ with operators in $\Psi_{1,0}^{k}$ are in $\Psi_{1 / 2,1 / 2}^{m+k-1 / 2}$. Observe that this condition is preserved when multiplying with symbols in $S_{1,0}^{0}$.

We are going to prove an estimate for operators $P_{0}$ which satisfy condition (2.2). Let $\|u\|_{(s)}$ be the usual Sobolev norm, let $\|u\|=\|u\|_{(0)}$ be the $L^{2}$ norm, and $\langle u, v\rangle$ the corresponding inner product. 
Proposition 2.2. Assume that $P=D_{t}+i F^{w}\left(t, x, D_{x}\right)$, with $F \in C\left(\mathbf{R}, S_{c l}^{1}\right)$ having real principal symbol $f$ satisfying condition (2.2). Then there exists $T_{0}>0$ such that if $0<T \leq T_{0}$ then we can choose a real valued symbol $b_{T}(t, x, \xi) \in L^{\infty}\left(\mathbf{R}, S_{1 / 2,1 / 2}^{1 / 2}\right)$ uniformly, with the property that $b_{T} \in S_{1 / 2,1 / 2}^{0}$ of first order uniformly, and

$$
\|u\|_{(-1 / 4)}^{2} \leq T \operatorname{Im}\left\langle P_{0} u, b_{T}^{w} u\right\rangle
$$

for $u(t, x) \in C_{0}^{\infty}\left(\mathbf{R} \times \mathbf{R}^{n}\right)$ having support where $|t| \leq T \leq T_{0}$.

Note that we have to change the multiplier $b_{T}$ when we change $T$, but that the multipliers are uniformly bounded in the symbol class. By the calculus, the conditions on $b_{T}$ are preserved when composing $b_{T}^{w}$ with symmetric operators in $L^{\infty}\left(\mathbf{R}, \Psi_{1,0}^{0}\right)$.

Remark 2.3. The estimate (2.3) can be perturbed with terms in $L^{\infty}\left(\mathbf{R}, S_{1,0}^{0}\right)$ in the symbol of $P_{0}$ for small enough $T$. Thus it can be microlocalized: if $\phi(x, \xi) \in S_{1,0}^{0}$ is real valued then we have

$$
\operatorname{Im}\left\langle P_{0} \phi^{w} u, b_{T}^{w} \phi^{w} u\right\rangle \leq \operatorname{Im}\left\langle P_{0} u, \phi^{w} b_{T}^{w} \phi^{w} u\right\rangle+C\|u\|_{(-1 / 4)}^{2}
$$

where $\phi^{w} b_{T}^{w} \phi^{w}$ satisfies the same conditions as $b_{T}^{w}$.

In fact, assume that $P_{0}=D_{t}+i f^{w}\left(t, x, D_{x}\right)+r^{w}\left(t, x, D_{x}\right)$ with $r \in L^{\infty}\left(\mathbf{R}, S_{1,0}^{0}\right)$. By conjugation with $E^{w}\left(t, x, D_{x}\right)$ where

$$
E(t, x, \xi)=\exp \left(-\int_{0}^{t} \operatorname{Im} r(s, x, \xi) d s\right) \in L^{\infty}\left(\mathbf{R}, S_{1,0}^{0}\right),
$$

we can reduce to the case when $\operatorname{Im} r \in L^{\infty}\left(\mathbf{R}, S_{1,0}^{-1}\right)$. We find that $b_{T}^{w}$ is replaced with $B_{T}^{w}=E^{w} b_{T}^{w} E^{w}$, which is real and satisfies the same conditions as $b_{T}^{w}$ since $E$ is real. Clearly, the estimate (2.3) can be perturbed with terms in $L^{\infty}\left(\mathbf{R}, S_{1,0}^{-1}\right)$ in the symbol expansion of $P_{0}$, and if $a(t, x, \xi) \in L^{\infty}\left(\mathbf{R}, S_{1,0}^{0}\right)$ is real valued, then

$$
\operatorname{Im}\left\langle a^{w} u, b_{T}^{w} u\right\rangle=\frac{1}{2 i}\left\langle\left[b_{T}^{w}, a^{w}\right] u, u\right\rangle \leq C\|u\|_{(-1 / 4)}^{2}
$$

since $b_{T} \in S_{1 / 2,1 / 2}^{0}$ of first order, $\forall t$. We also find that $\left[P_{0}, \phi^{w}\right] \cong\{f, \phi\}^{w}$ modulo $L^{\infty}\left(\mathbf{R}, \Psi_{1,0}^{-1}\right)$ where $\{f, \phi\} \in L^{\infty}\left(\mathbf{R}, S_{1,0}^{0}\right)$ is real valued. By using (2.5) with $a=$ $\{f, \phi\}$, we obtain that the estimate (2.3) is localizable.

Proof of Theorem 1.1. By using Darboux' theorem and the Malgrange Preparation Theorem, we may assume that the adjoint $P^{*}$ is equal to $P_{0}$ microlocally, where $P_{0}$ satisfies the conditions in Proposition 2.2 (see [10, Th. 21.3.6]). By using (2.3), a partition of unity, and the Cauchy-Schwarz inequality, we obtain $R \in S_{1,0}^{0}$, such that $x_{0} \notin \operatorname{sing} \operatorname{supp} R$ and

$$
\|u\|_{(-1 / 4)} \leq C\left\|P^{*} u\right\|_{(7 / 4-m)}+\left\|R^{w} u\right\|_{(-1 / 4)}
$$

for $u(x) \in C_{0}^{\infty}\left(\mathbf{R}^{n}\right)$ having support where $|x| \leq T_{0}$ is small enough. Now conjugation with $\left\langle D_{x}\right\rangle^{s}$ does not change the principal symbol of $P$. Thus, for any $s \in \mathbf{R}$ we may replace $-1 / 4$ by $s$ and $7 / 4$ by $s+2$ in (2.6) after changing $T_{0}$ and $R$. This gives the local solvability of $P$ with a loss of at most two derivatives, and finishes the proof of Theorem 1.1. 
Corollary 2.4. If $P \in \Psi_{c l}^{m}(M)$ is of principal type near $\left(x_{0}, \xi_{0}\right) \in T^{*} M$, satisfying condition $(\Psi)$ microlocally near $\left(x_{0}, \xi_{0}\right)$, then $P$ is microlocally solvable at $\left(x_{0}, \xi_{0}\right)$.

In order to prove Proposition 2.2 we shall need to make a "second microlocalization" using the specialized symbol classes of the Weyl calculus (see [10, Section 18.5]). Assume that $g_{x, \xi}(d x, d \xi)$ is a $\sigma$ temperate metric on $T^{*} \mathbf{R}^{n}$, and let $m$ be a $\sigma, g$ temperate. Let $S(m, g)$ be the class of symbols $a \in C^{\infty}\left(T^{*} \mathbf{R}^{n}\right)$ with the seminorms

$$
|a|_{j}^{g}(x, \xi)=\sup _{T_{i} \neq 0} \frac{\left|a^{(j)}\left(x, \xi, T_{1}, \ldots, T_{j}\right)\right|}{\prod_{1}^{j} g_{x, \xi}\left(T_{i}\right)^{1 / 2}} \leq C_{j} m(x, \xi) \quad \forall(x, \xi) \text { for } j \geq 0 .
$$

We shall use metrics which are conformal, they shall be on the form $g_{x, \xi}(d x, d \xi)=$ $H(x, \xi) g^{\sharp}(d x, d \xi)$ where $0<H(x, \xi) \leq 1$ and $g^{\sharp}$ is a constant symplectic metric: $\left(g^{\sharp}\right)^{\sigma}=g^{\sharp}$. In the following, we say that $m>0$ is a weight for a metric $g$ if $m$ is $\sigma$, $g$ temperate.

Definition 2.5. Let $m$ be a weight for the $\sigma$ temperate metric $g$. We say that $a \in S^{+}(m, g)$ if $|a|_{j}^{g} \leq C_{j} m$ for $j \geq 1$.

For example, $b \in S^{+}\left(1, g_{1 / 2,1 / 2}\right)$, with $g_{1 / 2,1 / 2}=\langle\xi\rangle|d x|^{2}+|d \xi|^{2} /\langle\xi\rangle$ at $(x, \xi)$, if and only if $b \in S_{1 / 2,1 / 2}^{0}$ of first order. After microlocalizing where $\langle\xi\rangle \cong h^{-1} \geq 1$ is constant, and doing a microlocal change of coordinates, we find that $S_{1,0}^{k}$ corresponds to $S\left(h^{-k}, h g^{\sharp}\right)$ and $S_{1 / 2,1 / 2}^{k}$ corresponds to $S\left(h^{-k}, g^{\sharp}\right)$ microlocally. Thus we may reduce to the case in the following result (see the proof of Proposition 2.2 in [7]).

Proposition 2.6. Assume that $P_{0}=D_{t}+i f^{w}\left(t, x, D_{x}\right)$, with real valued $f(t, x, \xi) \in$ $C\left(\mathbf{R}, S\left(h^{-1}, h g^{\sharp}\right)\right)$ satisfying condition $(2.2)$, here $0<h \leq 1$ and $g^{\sharp}=\left(g^{\sharp}\right)^{\sigma}$ are constant. Then there exists $T_{0}>0$, such that if $0<T \leq T_{0}$ there exist a weight $h \leq$ $H_{T} \leq 1$ for $g^{\sharp}$ and a real valued symbol $b_{T}(t, x, \xi) \in L^{\infty}\left(\mathbf{R}, S\left(H_{T}^{-1 / 2}, g^{\sharp}\right) \bigcap S^{+}\left(1, g^{\sharp}\right)\right)$ uniformly, so that

$$
h^{1 / 2} \int\|u\|^{2}(t) d t \leq C_{0} T \int \operatorname{Im}\left\langle P u, b_{T}^{w} u\right\rangle(t) d t
$$

for $u(t, x) \in C_{0}^{\infty}\left(\mathbf{R} \times \mathbf{R}^{n}\right)$ having support where $|t| \leq T \leq T_{0}$.

The conditions on $b_{T}$ means in $g^{\sharp}$ orthonormal coordinates that $\left|b_{T}\right| \leq C H_{T}^{-1 / 2}$ and $\left|\partial_{x}^{\alpha} \partial_{\xi}^{\beta} b_{T}\right| \leq C_{\alpha \beta}$ when $|\alpha|+|\beta| \geq 1$. As before, the estimate (2.7) can be perturbed with terms in $L^{\infty}\left(\mathbf{R}, S\left(1, h g^{\sharp}\right)\right)$ in the symbol of $P_{0}$ for small $T$ (with changed $b_{T}$ ), and it can be localized with respect to the metric $h g^{\sharp}$. Next, we shall state and prove the multiplier estimate that we are going to use for the proof of Proposition 2.6.

Let $\mathcal{B}=\mathcal{B}\left(L^{2}\left(\mathbf{R}^{n}\right)\right)$ be the set of bounded operators $L^{2}\left(\mathbf{R}^{n}\right) \mapsto L^{2}\left(\mathbf{R}^{n}\right)$. We say that $A(t) \in C(\mathbf{R}, \mathcal{B})$ if $A(t) \in \mathcal{B}$ for all $t \in \mathbf{R}$ and $t \mapsto A(t) u \in C\left(\mathbf{R}, L^{2}\left(\mathbf{R}^{n}\right)\right)$ for any $u \in L^{2}\left(\mathbf{R}^{n}\right)$. We shall consider the operator

$$
P=D_{t}+i F(t)
$$


where $F(t) \in C(\mathbf{R}, \mathcal{B})$. In the applications, we will have $F(t) \in C\left(\mathbf{R}\right.$, Op $\left.S\left(h^{-1}, h g^{\sharp}\right)\right)$ where $h$ is constant. But we shall also use multipliers which are not continuous in $t$. In the following, we let $\|u\|(t)$ be the $L^{2}$ norm of $u(t, x)$ in $\mathbf{R}^{n}$ for fixed $t$, and $\langle u, v\rangle(t)$ the corresponding inner product.

Definition 2.7. We say that $A(t)$ is in $L_{l o c}^{\infty}(\mathbf{R}, \mathcal{B})$ if $A(t) \in \mathcal{B}$ for any $t$, and $t \mapsto A(t) u$ is in $L_{l o c}^{\infty}\left(\mathbf{R}, L^{2}\left(\mathbf{R}^{n}\right)\right)$ for any $u \in L^{2}\left(\mathbf{R}^{n}\right)$, i.e., $t \mapsto\langle A(t) u, v\rangle$ is in $L_{l o c}^{\infty}(\mathbf{R})$ for any $u, v \in L^{2}\left(\mathbf{R}^{n}\right)$.

If $A(t) \in L_{l o c}^{\infty}(\mathbf{R}, \mathcal{B})$, then we find that $t \mapsto\langle A(t) u, u\rangle \in L_{l o c}^{\infty}(\mathbf{R})$ has weak derivative $\left\langle\frac{d}{d t} A(\cdot) u, u\right\rangle \in \mathcal{D}^{\prime}(\mathbf{R})$ for any $u \in \mathcal{S}\left(\mathbf{R}^{n}\right)$ given by

$$
\left\langle\frac{d}{d t} A(\cdot) u, u\right\rangle(\phi)=-\int\langle A(t) u, u\rangle \phi^{\prime}(t) d t, \quad \phi(t) \in C_{0}^{\infty}(\mathbf{R}) .
$$

It is also easy to see that if $u(t), v(t) \in C\left(\mathbf{R}, L^{2}\left(\mathbf{R}^{n}\right)\right)$ and $A(t) \in L_{l o c}^{\infty}(\mathbf{R}, \mathcal{B})$, then $t \mapsto\langle A(t) u(t), v(t)\rangle \in L_{\text {loc }}^{\infty}(\mathbf{R})$.

We shall use the following multiplier estimate (see also [13] and [15] for similar estimates).

Proposition 2.8. Let $P=D_{t}+i F(t)$ with $F(t) \in C(\mathbf{R}, \mathcal{B})$. Assume that $B(t)=$ $B^{*}(t) \in L_{\text {loc }}^{\infty}(\mathbf{R}, \mathcal{B})$ satisfies

$$
\operatorname{Re}\left\langle\frac{d}{d t} B(t) u, u\right\rangle+2 \operatorname{Re}\langle B(t) u, F(t) u\rangle \geq \operatorname{Re}\langle m(t) u, u\rangle \quad \text { in } \mathcal{D}^{\prime}(I) \quad \forall u \in C_{0}^{\infty}\left(\mathbf{R}^{n}\right)
$$

where $m(t) \in L_{\text {loc }}^{\infty}(\mathbf{R}, \mathcal{B})$ and $I \subseteq \mathbf{R}$ is an open interval. Then we have

$$
\int \operatorname{Re}\langle m(t) u(t), u(t)\rangle d t \leq 2 \int \operatorname{Im}\langle P u(t), B(t) u(t)\rangle d t
$$

for any $u \in C_{0}^{1}\left(I, C_{0}^{\infty}\left(\mathbf{R}^{n}\right)\right)$.

Proof. Since $B(t) \in \mathcal{B}$ is weakly measurable and locally bounded, we may for $u \in$ $C_{0}^{\infty}\left(\mathbf{R}^{n}\right)$ define the regularization

$$
\left\langle B_{\varepsilon}(t) u, u\right\rangle=\varepsilon^{-1} \int\langle B(s) u, u\rangle \phi((t-s) / \varepsilon) d s=\langle B u, u\rangle\left(\phi_{\varepsilon, t}\right) \quad \varepsilon>0
$$

where $\phi_{\varepsilon, r}(s)=\varepsilon^{-1} \phi((r-s) / \varepsilon)$ with $0 \leq \phi \in C_{0}^{\infty}(\mathbf{R})$ satisfying $\int \phi(t) d t=1$. Then $t \mapsto\left\langle B_{\varepsilon}(t) u, u\right\rangle$ is in $C^{\infty}(\mathbf{R})$ with derivative at $t=r$ equal to $\left\langle\frac{d}{d t} B_{\varepsilon}(r) u, u\right\rangle=$ $\frac{d}{d t}\langle B u, u\rangle\left(\phi_{\varepsilon, r}\right)$. Let $I_{0}$ be an open interval such that $I_{0} \Subset I$. Then for small enough $\varepsilon>0$ we find from condition (2.9) that

$$
\operatorname{Re}\left\langle\frac{d}{d t} B_{\varepsilon}(t) u, u\right\rangle+2 \operatorname{Re}\langle B u, F u\rangle\left(\phi_{\varepsilon, t}\right) \geq \operatorname{Re}\langle m u, u\rangle\left(\phi_{\varepsilon, t}\right) \quad t \in I_{0} \quad u \in C_{0}^{\infty}\left(\mathbf{R}^{n}\right) .
$$

In fact, $\phi_{\varepsilon, t} \geq 0$ and $\operatorname{supp} \phi_{\varepsilon, t} \in C_{0}^{\infty}(I)$ for small enough $\varepsilon$ when $t \in I_{0}$.

Now we define for $u \in C_{0}^{1}\left(I_{0}, C_{0}^{\infty}\left(\mathbf{R}^{n}\right)\right)$ and small enough $\varepsilon>0$

$$
M_{\varepsilon, u}(t)=\operatorname{Re}\left\langle B_{\varepsilon} u, u\right\rangle(t)=\varepsilon^{-1} \int\langle B(s) u(t), u(t)\rangle \phi((t-s) / \varepsilon) d s .
$$


By differentiating under the integral sign we obtain that $M_{\varepsilon, u}(t) \in C_{0}^{1}\left(I_{0}\right)$, with derivative $\frac{d}{d t} M_{\varepsilon, u}=\operatorname{Re}\left\langle\left(\frac{d}{d t} B_{\varepsilon}\right) u, u\right\rangle+2 \operatorname{Re}\left\langle B_{\varepsilon} u, \partial_{t} u\right\rangle$ since $B(t) \in L_{l o c}^{\infty}(\mathbf{R}, \mathcal{B})$. By integrating with respect to $t$, we obtain the vanishing average

$$
0=\int M_{\varepsilon, u}(t) d t=\int \operatorname{Re}\left\langle\left(\frac{d}{d t} B_{\varepsilon}\right) u, u\right\rangle d t+\int 2 \operatorname{Re}\left\langle B_{\varepsilon} u, \partial_{t} u\right\rangle d t
$$

when $u \in C_{0}^{1}\left(I_{0}, C_{0}^{\infty}\left(\mathbf{R}^{n}\right)\right)$. Since $\partial_{t} u=i P u+F u$ we obtain from (2.11) and (2.13) that

$$
\begin{aligned}
0 \geq \iint(\operatorname{Re}\langle m(s) u(t), u(t)\rangle+ & 2 \operatorname{Re}\langle B(s) u(t), i P u(t)\rangle \\
& +\operatorname{Re}\langle B(s) u(t),(F(t)-F(s)) u(t)\rangle) \phi_{\varepsilon, t}(s) d s d t .
\end{aligned}
$$

By letting $\varepsilon \rightarrow 0$ we obtain by dominated convergence that

$$
0 \geq \int \operatorname{Re}\langle m(t) u(t), u(t)\rangle+2 \operatorname{Re}\langle B(t) u(t), i P u(t)\rangle d t
$$

since $F(t) \in C(\mathbf{R}, \mathcal{B}), u \in C_{0}^{1}\left(I_{0}, C_{0}^{\infty}\left(\mathbf{R}^{n}\right)\right), m(t)$ and $B(t)$ are uniformly bounded in $\mathcal{B}$ when $t \in \operatorname{supp} u$. Now $2 \operatorname{Re}\langle B u, i P u\rangle=-2 \operatorname{Im}\langle P u, B u\rangle$, thus we obtain (2.10) for $u \in C_{0}^{1}\left(I_{0}, C_{0}^{\infty}\left(\mathbf{R}^{n}\right)\right)$. Since $I_{0}$ is an arbitrary open subinterval with compact closure in $I$, this completes the proof of the proposition.

Now we can reduce the proof of Proposition 2.6 to the construction of a pseudo$\operatorname{sign} B=b^{w}$ in a fixed interval.

Proposition 2.9. Assume that $f \in C\left(\mathbf{R}, S\left(h^{-1}, h g^{\sharp}\right)\right)$ is a real valued symbol satisfying condition $(\bar{\Psi})$ given by $(2.2)$, here $0<h \leq 1$ and $g^{\sharp}=\left(g^{\sharp}\right)^{\sigma}$ are constant. Then there exist a positive constant $c_{0}$, a weight $h \leq H_{1} \leq 1$ for $g^{\sharp}$, real valued symbols $b(t, x, \xi) \in L^{\infty}\left(\mathbf{R}, S\left(H_{1}^{-1 / 2}, g^{\sharp}\right)+S^{+}\left(1, g^{\sharp}\right)\right)$ and $\mu(t, x, \xi) \in L^{\infty}\left(\mathbf{R}, S\left(1, g^{\sharp}\right)\right)$ such that for any $u(x) \in C_{0}^{\infty}\left(\mathbf{R}^{n}\right)$ we have

$$
\left\{\begin{array}{l}
\left\langle\partial_{t}\left(b^{w}\right) u, u\right\rangle \geq\left\langle\mu^{w} u, u\right\rangle \geq c_{0} h^{1 / 2}\|u\|^{2} \\
\operatorname{Re}\left\langle b^{w} f^{w} u, u\right\rangle \geq-\left\langle\mu^{w} u, u\right\rangle / c_{0}
\end{array} \quad \text { in } \mathcal{D}^{\prime}(\mathbf{R}) \text { when }|t|<1 .\right.
$$

Here $c_{0}$, and the seminorms of $b$ and $m$ only depend on the seminorms of $f$ in $S\left(h^{-1}, h g^{\sharp}\right)$ for $|t| \leq 1$.

Proof of Proposition 2.6. By doing a dilation $s=t / T$, we find that $P$ transforms into $T^{-1} P_{T}=T^{-1}\left(D_{s}+i T f_{T}^{w}\left(s, x, D_{x}\right)\right)$, where $f_{T}(s, x, \xi)=f(T s, x, \xi)$ satisfies the conditions in Proposition 2.9 uniformly in $T$ when $0<T \leq 1$. Thus we obtain real $b_{T}, \mu_{T}$ and $c_{0}$ such that when $|s|<1$ we have

$$
\left\{\begin{array}{l}
\left\langle\partial_{s}\left(b_{T}^{w}\right) u, u\right\rangle \geq\left\langle\mu_{T}^{w} u, u\right\rangle \geq c_{0} h^{1 / 2}\|u\|^{2} \\
\operatorname{Re}\left\langle b_{T}^{w} f_{T}^{w} u, u\right\rangle \geq-\left\langle\mu_{T}^{w} u, u\right\rangle / c_{0}
\end{array} \quad \text { in } \mathcal{D}^{\prime}(\mathbf{R})\right.
$$

for $u \in C_{0}^{\infty}\left(\mathbf{R}^{n}\right)$. This implies that

$$
\begin{aligned}
\left\langle\partial_{s} b_{T}^{w}\left(s, x, D_{x}\right) u, u\right\rangle+2 \operatorname{Re}\langle T & \left.f_{T}^{w}\left(s, x, D_{x}\right) u, b_{T}^{w}\left(s, x, D_{x}\right) u\right\rangle \\
& \geq\left(1-2 T / c_{0}\right)\left\langle\mu_{T}^{w}\left(s, x, D_{x}\right) u, u\right\rangle \quad \text { in } \mathcal{D}^{\prime}(]-1,1[)
\end{aligned}
$$


for $u \in C_{0}^{\infty}\left(\mathbf{R}^{n}\right)$. Thus, for $T \leq c_{0} / 4$ we obtain by using Proposition 2.8 with $P_{T}=D_{s}+i T f_{T}^{w}\left(s, x, D_{x}\right), B(s)=b_{T}^{w}\left(s, x, D_{x}\right)$ and $m(s)=\mu_{T}^{w}\left(s, x, D_{x}\right)$ that

$$
c_{0} h^{1 / 2} \int\|u\|^{2} d s \leq \int\left\langle\mu_{T}^{w} u, u\right\rangle d s \leq 4 \int \operatorname{Im}\left\langle P_{T} u, b_{T}^{w} u\right\rangle(s) d s
$$

if $u \in C_{0}^{\infty}\left(\mathbf{R} \times \mathbf{R}^{n}\right)$ has support where $|s|<1$. Finally, we obtain that

$$
c_{0} h^{1 / 2} \int\|u\|^{2} d t \leq 4 T \int \operatorname{Im}\left\langle P u, \widetilde{b}_{T}^{w} u\right\rangle(t) d t
$$

with $\widetilde{b}_{T}(t, x, \xi)=b_{T}(t / T, x, \xi)$ for $u \in C_{0}^{\infty}\left(\mathbf{R} \times \mathbf{R}^{n}\right)$ has support where $|t|<T \leq$ $c_{0} / 4$.

It remains to prove Proposition 2.9, which will be done in Section 5 .

\section{Symbol Classes and Weights}

Next, we shall define the symbol classes we shall use. In the following, we shall denote $(x, \xi)$ by $w \in T^{*} \mathbf{R}^{n}$, and we shall assume that $f \in C\left(\mathbf{R}, S\left(h^{-1}, h g^{\sharp}\right)\right)$ satisfies condition $(\bar{\Psi})$ given by $(2.2)$, here $0<h \leq 1$ and $g^{\sharp}=\left(g^{\sharp}\right)^{\sigma}$ are constant. We shall only consider the values of $f(t, w)$ when $|t| \leq 1$, thus for simplicity we let $f(t, w)=f(1, w)$ when $t \geq 1$ and $f(t, w)=f(-1, w)$ when $t \leq-1$. In the following, the results will be uniform in the sense that they will only depend on the seminorms of $f$ in $S\left(h^{-1}, h g^{\sharp}\right)$.

First, we shall define the signed distance function $\delta_{0}(t, w)$ in $T^{*} \mathbf{R}^{n}$ for fixed $t \in \mathbf{R}$, with the property that $t \mapsto \delta_{0}(t, w)$ is non-decreasing and $\delta_{0} f \geq 0$. Let

$$
\begin{aligned}
& X_{+}=\left\{(t, w) \in \mathbf{R} \times T^{*} \mathbf{R}^{n}: \exists s \leq t, f(s, w)>0\right\} \\
& X_{-}=\left\{(t, w) \in \mathbf{R} \times T^{*} \mathbf{R}^{n}: \exists s \geq t, f(s, w)<0\right\}
\end{aligned}
$$

We have that $X_{ \pm}$are open in $\mathbf{R} \times T^{*} \mathbf{R}^{n}$, and by condition $(\bar{\Psi})$ we obtain that $X_{-} \bigcap X_{+}=\emptyset$ and $\pm f \geq 0$ on $X_{ \pm}$. Let $X_{0}=\mathbf{R} \times T^{*} \mathbf{R}^{n} \backslash\left(X_{+} \cup X_{-}\right)$, which is closed in $\mathbf{R} \times T^{*} \mathbf{R}^{n}$, by the definition of $X_{ \pm}$we have $f=0$ on $X_{0}$. Let

$$
d_{0}\left(t_{0}, w_{0}\right)=\inf \left\{g^{\sharp}\left(w_{0}-z\right)^{1 / 2}:\left(t_{0}, z\right) \in X_{0}\right\}
$$

which is the $g^{\sharp}$ distance in $T^{*} \mathbf{R}^{n}$ to $X_{0}$ for fixed $t_{0}$, it is identically equal to $+\infty$ in the case that $X_{0} \bigcap\left\{t=t_{0}\right\}=\emptyset$.

Definition 3.1. We say that $w \mapsto a(w)$ is Lipschitz continuous on $T^{*} \mathbf{R}^{n}$ with respect to the metric $g^{\sharp}$ if

$$
\sup _{w \neq z \in T^{*} \mathbf{R}^{n}}|a(w)-a(z)| / g^{\sharp}(w-z)^{1 / 2}=C<\infty
$$

and then $C$ is the Lipschitz constant of $a$. We shall denote by $\operatorname{Lip}\left(T^{*} \mathbf{R}^{n}\right)$ the Lipschitz continuous functions on $T^{*} \mathbf{R}^{n}$ with respect to the metric $g^{\sharp}$. 
By using the triangle inequality and taking the infimum over $z$ we find that $w \mapsto d_{0}(t, w)$ is Lipschitz continuous with respect to the metric $g^{\sharp}$ with Lipschitz constant equal to 1 , for those $t$ when it is not identically equal to $\infty$.

Definition 3.2. We define the sign of $f$ by $\operatorname{sgn}(f)= \pm 1$ on $X_{ \pm}$and $\operatorname{sgn}(f)=0$ on $X_{0}$, then $t \rightarrow \operatorname{sgn}(f)(t, w)$ is non-decreasing and $\operatorname{sgn}(f) \cdot f \geq 0$. We define the signed distance function $\delta_{0}$ by

$$
\delta_{0}(t, w)=\operatorname{sgn}(f)(t, w) \min \left(d_{0}(t, w), h^{-1 / 2}\right) .
$$

By the definition we have that $\left|\delta_{0}\right| \leq h^{-1 / 2}$ and $\left|\delta_{0}\right|=d_{0}$ when $\left|\delta_{0}\right|<h^{-1 / 2}$. The signed distance function has the following properties.

Remark 3.3. The signed distance function $w \mapsto \delta_{0}(t, w)$ given by Definition 3.2 is Lipschitz continuous with respect to the metric $g^{\sharp}$ with Lipschitz constant equal to 1. We also find that $\delta_{0}(t, w) f(t, w) \geq 0$ and $t \mapsto \delta_{0}(t, w)$ is non-decreasing.

In fact, since $\delta_{0}=0$ on $X_{0}$ it suffices to show the Lipschitz continuity of $w \mapsto$ $\delta_{0}(t, w)$ on $X_{+}$and $X_{-}$, which follows from the Lipschitz continuity of $w \mapsto d_{0}(t, w)$. Since $(t, w) \in X_{+}$implies $(s, w) \in X_{+}$for $s \geq t$ and $(t, w) \in X_{-}$implies $(s, w) \in X_{-}$ for $s \leq t$, it is easy to see that $t \mapsto \delta_{0}(t, w)$ is non-decreasing. Since $t \mapsto \delta_{0}(t, w)$ is non-decreasing and bounded, it is a regulated function. This means that the left and right limits $\delta_{0}(t \pm, w)=\lim _{0<\varepsilon \rightarrow 0} \delta_{0}(t \pm \varepsilon, w)$ exist for any $(t, w)$ (see [8]).

In the following, we shall omit the parameter $t$, and denote $f^{\prime}=\partial_{w} f$ and $f^{\prime \prime}=f^{(2)}$, where the differentiation is in the $w$ variables only. We shall also in the following assume that we have choosen $g^{\sharp}$ orthonormal coordinates so that $g^{\sharp}(d w)=$ $|d w|^{2}$. We shall use the norms $\left|f^{\prime}\right|_{g^{\sharp}}=\left|f^{\prime}\right|$ and $\left\|f^{\prime \prime}\right\|_{g^{\sharp}}=\left\|f^{\prime \prime}\right\|$, but omit the index $g^{\sharp}$.

Definition 3.4. Let

$$
H_{1}^{-1 / 2}=1+\left|\delta_{0}\right|+\frac{\left|f^{\prime}\right|}{\left\|f^{\prime \prime}\right\|+h^{1 / 4}\left|f^{\prime}\right|^{1 / 2}+h^{1 / 2}}
$$

and $G_{1}=H_{1} g^{\sharp}$ the corresponding metric.

Since $\left|f^{\prime}\right| /\left(\left\|f^{\prime \prime}\right\|+h^{1 / 4}\left|f^{\prime}\right|^{1 / 2}+h^{1 / 2}\right)$ is continuous in $(t, w)$ we find that $t \mapsto$ $H_{1}^{1 / 2}(t, w)$ is a regulated function. We also have

$$
1 \leq H_{1}^{-1 / 2} \leq 1+\left|\delta_{0}\right|+h^{-1 / 4}\left|f^{\prime}\right|^{1 / 2} \leq C h^{-1 / 2}
$$

since $\left|f^{\prime}\right| \leq C_{1} h^{-1 / 2}$ and $\left|\delta_{0}\right| \leq h^{-1 / 2}$. Moreover, $\left|f^{\prime}\right| \leq H_{1}^{-1 / 2}\left(\left\|f^{\prime \prime}\right\|+h^{1 / 4}\left|f^{\prime}\right|^{1 / 2}+\right.$ $h^{1 / 2}$ ) so the Cauchy-Schwarz inequality gives

$$
\left|f^{\prime}\right| \leq 2\left\|f^{\prime \prime}\right\| H_{1}^{-1 / 2}+3 h^{1 / 2} H_{1}^{-1} \leq C H_{1}^{-1 / 2} .
$$

In the case $1+\left|\delta_{0}\left(w_{0}\right)\right| \leq H_{1}^{-1 / 2}\left(w_{0}\right) / 2$ we have

$$
H_{1}^{-1 / 2}\left(w_{0}\right) \leq 2\left|f^{\prime}\left(w_{0}\right)\right| /\left(\left\|f^{\prime \prime}\left(w_{0}\right)\right\|+h^{1 / 4}\left|f^{\prime}\left(w_{0}\right)\right|^{1 / 2}+h^{1 / 2}\right),
$$


then we find

$$
\begin{aligned}
& \left\|f^{\prime \prime}\left(w_{0}\right)\right\| \leq 2 H_{1}^{1 / 2}\left(w_{0}\right)\left|f^{\prime}\left(w_{0}\right)\right| \quad \text { and } \\
& h^{1 / 2} \leq 4 H_{1}\left(w_{0}\right)\left|f^{\prime}\left(w_{0}\right)\right| .
\end{aligned}
$$

By Proposition 3.6 below the metric $G_{1}$ is $\sigma$ temperate. The denominator

$$
D=\left\|f^{\prime \prime}\right\|+h^{1 / 4}\left|f^{\prime}\right|^{1 / 2}+h^{1 / 2}
$$

in (3.4) may seem strange, but it has the following natural explanation which we owe to Nicolas Lerner [18]. We have $F=h^{-1 / 2} f \in S\left(h^{-3 / 2}, g\right)$, and the largest $H_{2} \leq 1$ for which $F \in S\left(H_{2}^{-3 / 2}, H_{2} g^{\sharp}\right)$ is given by

$$
H_{2}^{-1 / 2} \cong 1+|F|^{1 / 3}+\left|F^{\prime}\right|^{1 / 2}+\left\|F^{\prime \prime}\right\|=1+h^{-1 / 6}|f|^{1 / 3}+h^{-1 / 4}\left|f^{\prime}\right|^{1 / 2}+h^{-1 / 2}\left\|f^{\prime \prime}\right\|
$$

modulo bounded factors. We obtain that $H_{2}^{-1 / 2} \cong 1+h^{-1 / 4}\left|f^{\prime}\right|^{1 / 2}+h^{-1 / 2}\left\|f^{\prime \prime}\right\|=$ $D h^{-1 / 2}$ and $H_{1}^{-1 / 2} \cong 1+\left|\delta_{0}\right|+\left|F^{\prime}\right| H_{2}^{1 / 2} \leq C H_{2}^{-1 / 2}$ in a $G_{2}$ neighborhood of $f^{-1}(0)$, and $H_{2}^{-1 / 2} \cong H_{1}^{-1 / 2}+\left\|F^{\prime \prime}\right\|$ in a $G_{1}$ neighborhood of $f^{-1}(0)$.

Definition 3.5. Let

$$
M=|f|+\left|f^{\prime}\right| H_{1}^{-1 / 2}+\left\|f^{\prime \prime}\right\| H_{1}^{-1}+h^{1 / 2} H_{1}^{-3 / 2} .
$$

Then we have $h^{1 / 2} \leq M \leq c h^{-1}$, and $M$ has the following properties.

Proposition 3.6. We find that $G_{1}$ is $\sigma$ temperate such that $G_{1}=H_{1}^{2} G_{1}^{\sigma}$ and

$$
H_{1}(w) \leq C_{0} H_{1}\left(w_{0}\right)\left(1+H_{1}(w) g^{\sharp}\left(w-w_{0}\right)\right) \leq C_{0} H_{1}\left(w_{0}\right)\left(1+g^{\sharp}\left(w-w_{0}\right)\right) .
$$

We also have that $M$ is a weight for $G_{1}$ such that

$$
M(w) \leq C_{1} M\left(w_{0}\right)\left(1+H_{1}\left(w_{0}\right) g^{\sharp}\left(w-w_{0}\right)\right)^{3 / 2} \leq C_{1} M\left(w_{0}\right)\left(1+g^{\sharp}\left(w-w_{0}\right)\right)^{3 / 2}
$$

and $f \in S\left(M, G_{1}\right)$.

Observe that $H_{T}$ is a weight for $g^{\sharp}$ since $G_{T} \leq g^{\sharp}$. The advantage of using the metric $G_{1}$ is that in the case $H_{1} \ll 1$ in a $G_{1}$ neighborhood of the sign changes, we obtain that $\left|f^{\prime}\right| \geq c h^{1 / 2}$ is a weight for $G_{1}, \delta_{0} \in S\left(H_{1}^{-1 / 2}, G_{1}\right)$ and the curvature of $f^{-1}(0)$ is bounded by $C H_{1}^{1 / 2}$ (see Remark 3.7 and Proposition 3.8 below).

Proof of Proposition 3.6. Now, if $G_{1, w_{0}}\left(w-w_{0}\right) \geq c$ then $g^{\sharp}\left(w-w_{0}\right)=\left|w-w_{0}\right|^{2} \geq$ $c H_{1}^{-1}\left(w_{0}\right)$ which immediately gives (3.12). Thus it suffices to show that $G_{1}$ is slowly varying in order to prove (3.12).

First we consider the case $1+d_{0}\left(w_{0}\right) \geq H_{1}^{-1 / 2}\left(w_{0}\right) / 2$. Then we find by the uniform Lipschitz continuity of $w \mapsto d_{0}(w)$ that

$$
H_{1}^{-1 / 2}(w) \geq 1+d_{0}(w) \geq 1+d_{0}\left(w_{0}\right)-H_{1}^{-1 / 2}\left(w_{0}\right) / 6 \geq H_{1}^{-1 / 2}\left(w_{0}\right) / 3
$$

when $\left|w-w_{0}\right| \leq H_{1}^{-1 / 2}\left(w_{0}\right) / 6$, which gives slow variation in this case. 
In the case $1+d_{0}\left(w_{0}\right) \leq H_{1}^{-1 / 2}\left(w_{0}\right) / 2$ we obtain from Taylor's formula and (3.8) that

$$
\begin{aligned}
\left|f^{\prime}(w)\right| \leq\left|f^{\prime}\left(w_{0}\right)\right|+ & \varepsilon H_{1}^{-1 / 2}\left(w_{0}\right)\left\|f^{\prime \prime}\left(w_{0}\right)\right\|+C \varepsilon^{2} h^{1 / 2} H_{1}^{-1}\left(w_{0}\right) \\
& \leq\left(1+2 \varepsilon+4 C \varepsilon^{2}\right)\left|f^{\prime}\left(w_{0}\right)\right| \quad \text { when }\left|w-w_{0}\right| \leq \varepsilon H_{1}^{-1 / 2}\left(w_{0}\right)
\end{aligned}
$$

and similarly $\left|f^{\prime}(w)\right| \geq\left(1-2 \varepsilon-4 C \varepsilon^{2}\right)\left|f^{\prime}\left(w_{0}\right)\right|$. Thus we obtain that

$$
1-C^{\prime} \varepsilon \leq\left|f^{\prime}(w)\right| /\left|f^{\prime}\left(w_{0}\right)\right| \leq 1+C^{\prime} \varepsilon \quad \text { when }\left|w-w_{0}\right| \leq \varepsilon H_{1}^{-1 / 2}\left(w_{0}\right)
$$

in the case $H_{1}^{1 / 2} \leq 1 / 4$ and $\left|\delta_{0}\right| \leq H_{1}^{-1 / 2} / 4$ at $w_{0}$. Taylor's formula and (3.9) gives

$$
\left\|f^{\prime \prime}(w)\right\| \leq\left\|f^{\prime \prime}\left(w_{0}\right)\right\|+C \varepsilon H_{1}^{-1 / 2}\left(w_{0}\right) h^{1 / 2} \leq\left\|f^{\prime \prime}\left(w_{0}\right)\right\|+4 C \varepsilon H_{1}^{1 / 2}\left(w_{0}\right)\left|f^{\prime}\left(w_{0}\right)\right|
$$

when $\left|w-w_{0}\right| \leq \varepsilon H_{1}^{-1 / 2}\left(w_{0}\right)$. Thus we obtain from (3.7) and (3.14) that

$$
H_{1}^{1 / 2}(w) \leq\left\|f^{\prime \prime}(w)\right\|\left|f^{\prime}\right|^{-1}(w)+h^{1 / 4}\left|f^{\prime}\right|^{-1 / 2}(w)+h^{1 / 2}\left|f^{\prime}\right|^{-1}(w) \leq 3 H_{1}^{1 / 2}\left(w_{0}\right)
$$

when $\left|w-w_{0}\right| \leq \varepsilon H_{1}^{-1 / 2}\left(w_{0}\right)$ and $\varepsilon$ is small enough, which gives the slow variation.

Next, we prove (3.13). Taylor's formula gives as before that

$$
\left\|f^{(k)}(w)\right\| \leq C\left(\sum_{j=0}^{2-k} \| f^{(k+j)}\left(w_{0}\right)||\left|w-w_{0}\right|^{j}+h^{1 / 2}\left|w-w_{0}\right|^{3-k}\right) \quad 0 \leq k \leq 2 .
$$

Thus we obtain from Definition 3.5 that

$$
M(w) \leq C \sum_{k=0}^{2}\left\|f^{(k)}\left(w_{0}\right)\right\|\left(\left|w-w_{0}\right|+H_{1}^{-1 / 2}(w)\right)^{k}+C h^{1 / 2}\left(\left|w-w_{0}\right|+H_{1}^{-1 / 2}(w)\right)^{3} .
$$

We obtain from $(3.12)$ that $H_{1}^{-1 / 2}(w) \leq C\left(H_{1}^{-1 / 2}\left(w_{0}\right)+\left|w-w_{0}\right|\right)$. This gives

$$
\begin{aligned}
& M(w) \leq C \sum_{k=0}^{2}\left\|f^{(k)}\left(w_{0}\right)\right\| H_{1}^{-k / 2}\left(w_{0}\right)\left(1+H_{1}^{1 / 2}\left(w_{0}\right)\left|w-w_{0}\right|\right)^{k} \\
& +C h^{1 / 2} H_{1}^{-3 / 2}\left(w_{0}\right)\left(1+H_{1}^{1 / 2}\left(w_{0}\right)\left|w-w_{0}\right|\right)^{3} \leq C^{\prime} M\left(w_{0}\right)\left(1+H_{1}^{1 / 2}\left(w_{0}\right)\left|w-w_{0}\right|\right)^{3}
\end{aligned}
$$

and (3.13).

It is clear from the definition of $M$ that $\left\|f^{(k)}\right\| \leq M H_{1}^{k / 2}$ when $k \leq 2$, and when $k \geq 3$ we have

$$
\left\|f^{(k)}\right\| \leq C_{k} h^{\frac{k-2}{2}} \leq C_{k}^{\prime} h^{1 / 2} H_{1}^{\frac{k-3}{2}} \leq C_{k}^{\prime} M H_{1}^{\frac{k}{2}}
$$

since $h \leq C H_{1}$ by $(3.5)$ and $h^{1 / 2} H_{1}^{-3 / 2} \leq M$. This completes the proof.

Observe that $f \in S\left(M, H_{1} g^{\sharp}\right)$ for any choice of $H_{1} \geq$ ch in Definition 3.5, we do not use any other property of $H_{1}$. 
Remark 3.7. When $1+\left|\delta_{0}\left(w_{0}\right)\right| \leq H_{1}^{-1 / 2}\left(w_{0}\right) / 2$ we find that $\left|f^{\prime}\left(w_{0}\right)\right| \geq h^{1 / 2} / 4$ and

$$
1 / C \leq\left|f^{\prime}(w)\right| / \mid f^{\prime}\left(w_{0} \mid \leq C \quad \text { for }\left|w-w_{0}\right| \leq \varepsilon H_{1}^{-1 / 2}\left(w_{0}\right) .\right.
$$

We also have that $f^{\prime} \in S\left(\left|f^{\prime}\right|, G_{1}\right)$, i.e.,

$$
\left|f^{(k)}\left(w_{0}\right)\right| \leq C_{k}\left|f^{\prime}\left(w_{0}\right)\right| H_{1}^{\frac{k-1}{2}}\left(w_{0}\right) \quad \text { for } k \geq 1
$$

when $1+\left|\delta_{0}\left(w_{0}\right)\right| \leq H_{1}^{-1 / 2}\left(w_{0}\right) / 2$.

In fact, (3.17) is trivial if $k=1$, follows from (3.8) for $k=2$, and when $k \geq 3$ we have

$$
\left|f^{(k)}\left(w_{0}\right)\right| \leq C_{k} h^{\frac{k-2}{2}} \leq 4 C_{k}\left|f^{\prime}\right| H_{1} h^{\frac{k-3}{2}} \leq C_{k}^{\prime}\left|f^{\prime}\right| H_{1}^{\frac{k-1}{2}}
$$

by (3.5) and (3.9).

Proposition 3.8. Let $H_{1}^{-1 / 2}$ be given by Definition 3.4 for $f \in S\left(h^{-1}, h g^{\sharp}\right)$. There exists $\kappa_{1}>0$ so that if $\left|\delta_{0}\left(w_{0}\right)\right| \leq \kappa_{1} H_{1}^{-1 / 2}\left(w_{0}\right), H_{1}^{1 / 2}\left(w_{0}\right) \leq \kappa_{1}$ and

$$
\partial_{w_{1}} f\left(w_{0}\right) \geq \kappa_{1}\left|f^{\prime}\left(w_{0}\right)\right|
$$

then there exists $c_{1}>0$ such that

$$
\begin{aligned}
& f(w)=\alpha_{1}(w)\left(w_{1}-\beta\left(w^{\prime}\right)\right) \\
& \delta_{0}(w)=\alpha_{0}(w)\left(w_{1}-\beta\left(w^{\prime}\right)\right)
\end{aligned}
$$

when $\left|w-w_{0}\right| \leq c_{1} H_{1}^{-1 / 2}\left(w_{0}\right)$. Here $0<c_{1} \leq \alpha_{0} \in S\left(1, G_{1}\right), c_{1}\left|f^{\prime}\right| \leq \alpha_{1} \in$ $S\left(\left|f^{\prime}\right|, G_{1}\right)$ and $\beta \in S\left(H_{1}^{-1 / 2}, G_{1}\right)$ only depends on $w^{\prime}, w=\left(w_{1}, w^{\prime}\right)$.

Proof. We shall choose coordinates so that $w_{0}=0$, and put $H_{1}=H_{1}(0)$. Since $H_{1}^{1 / 2} \leq \kappa_{1}$ and $\delta_{0}(0)=0$ we find from (3.7) and the slow variation that

$$
\| f^{\prime \prime}(w)||\left|f^{\prime}(w)\right|^{-1}+h^{1 / 4}\left|f^{\prime}(w)\right|^{-1 / 2} \leq C H_{1}^{1 / 2}
$$

when $|w| \leq \varepsilon H_{1}^{-1 / 2}$ for sufficiently small $\varepsilon$ and $\kappa_{1}$. Remark 3.7 gives that $\left|f^{\prime}(w)\right| \leq$ $C\left|f^{\prime}(0)\right|$ when $|w| \leq \varepsilon H_{1}^{-1 / 2}$ for small $\varepsilon$. We find from (3.21) that

$$
\partial_{w_{1}} f(w) \geq \partial_{w_{1}} f(0)-C^{\prime} \varepsilon\left|f^{\prime}(0)\right| \geq \kappa_{1}\left|f^{\prime}(0)\right| / 2 \geq c h^{1 / 2}
$$

when $|w| \leq \varepsilon H_{1}^{-1 / 2}$ and $\varepsilon>0$ is small enough. Thus, by the implicit function theorem we can solve

$$
f(w)=0 \Longleftrightarrow w_{1}=\beta\left(w^{\prime}\right) \quad \text { when }|w| \leq \varepsilon H_{1}^{-1 / 2}
$$

for sufficiently small $\varepsilon>0$. We find that $\beta(0)=0,\left|\beta^{\prime}\right|=\left|\partial_{w^{\prime}} f\right| /\left|\partial_{w_{1}} f\right|=\mathcal{O}(1)$ and

$$
\left|\beta^{\prime \prime}\right| \leq C\left(\left|\partial_{w_{1}}^{2} f\left\|\left.\beta^{\prime}\right|^{2}+2\left|\partial_{w^{\prime}} \partial_{w_{1}} f\right||d \beta|+\right\| \partial_{w^{\prime}}^{2} f \|\right) /\left|\partial_{w_{1}} f\right|=\mathcal{O}\left(H_{1}^{1 / 2}\right)\right.
$$

when $w_{1}=\beta\left(w^{\prime}\right)$ and $|w| \leq \varepsilon H_{1}^{-1 / 2}$. 
Assume by induction that $\left|\partial^{\alpha} \beta\right| \leq C_{k} H_{1}^{(|\alpha|-1) / 2}$ when $|w| \leq \varepsilon H_{1}^{-1 / 2}(0)$ for $|\alpha|<$ $N$, where $N \geq 3$. Then we find for $|\alpha|=N$

$\partial^{\alpha} \beta=-\left(\sum c_{\gamma} \partial_{w_{1}}^{k} \partial_{w^{\prime}}^{\gamma_{0}} f \prod_{j=1}^{k} \partial_{w^{\prime}}^{\gamma_{j}} \beta+\partial_{w^{\prime}}^{\alpha} f\right) / \partial_{w_{1}} f \quad$ when $w_{1}=\beta\left(w^{\prime}\right)$ and $|w| \leq \varepsilon H_{1}^{-1 / 2}$ where the sum is over $k \geq 2$ and $\sum_{j=1}^{k} \gamma_{j}+\gamma_{0}=\alpha$; or $k=1, \gamma_{0} \neq 0$ and $\gamma_{0}+\gamma_{1}=\alpha$. In any case, we obtain that $k+\left|\gamma_{0}\right| \geq 2$.

When $|\alpha|=N \geq 3$ we find $\left|\partial^{\alpha} f / \partial_{w_{1}} f\right| \leq C_{N} h^{(|\alpha|-2) / 2} /\left|\partial_{w_{1}} f\right| \leq C_{N}^{\prime} H_{1}^{(|\alpha|-1) / 2}$, since we have $h \leq C H_{1}$ by (3.5) and $h^{1 / 2} /\left|\partial_{w_{1}} f\right| \leq C H_{1}$ by (3.21) when $|w| \leq$ $\varepsilon H_{1}^{-1 / 2}$. Similarly, for $k+\left|\gamma_{0}\right| \geq 2$ we find by the induction hypothesis that

$$
\left|\partial_{w_{1}}^{k} \partial_{w^{\prime}}^{\gamma_{0}} f \prod_{j=1}^{k} \partial_{w^{\prime}}^{\gamma_{j}} \beta / \partial_{w_{1}} f\right| \leq C_{N} H_{1}^{(|\alpha|-1) / 2}
$$

when $|w| \leq \varepsilon H_{1}^{-1 / 2}$. In fact, the case $k+\left|\gamma_{0}\right| \geq 3$ works as before since $\sum_{j=0}^{k}\left|\gamma_{j}\right|=$ $|\alpha|$, and when $k+\left|\gamma_{0}\right|=2$ we use that $\left\|f^{\prime \prime}(w)\right\| /\left|\partial_{w_{1}} f(w)\right| \leq C H_{1}^{1 / 2}$. This completes the induction argument.

Now by using Taylor's formula we find $f(w)=\alpha(w)\left(w_{1}-\beta\left(w^{\prime}\right)\right)$ where

$$
\alpha(w)=\int_{0}^{1} \partial_{w_{1}} f\left(\theta w_{1}+(1-\theta) \beta\left(w^{\prime}\right), w^{\prime}\right) d \theta \quad|w| \leq \varepsilon H_{1}^{-1 / 2} .
$$

Thus $\alpha(w) \cong\left|f^{\prime}(0)\right|$ since $\left|\beta\left(w^{\prime}\right)\right| \leq C \varepsilon H_{1}^{-1 / 2}$ when $|w| \leq \varepsilon H_{1}^{-1 / 2}$. Now we have $\partial_{w_{1}} f \in S\left(|d f|, G_{1}\right)$ by Remark 3.7, so $\alpha(w)=f_{0}\left(w, \beta\left(w^{\prime}\right)\right)$ for some $f_{0} \in S\left(|d f|, G_{1}\right)$ when $|w| \leq \varepsilon H_{1}^{-1 / 2}$. Thus differentiation gives

$$
\left|\partial^{\gamma} \alpha\right| \leq C \sum_{\sum_{j=1}^{k} \gamma_{j}+\gamma_{0}=\gamma}\left|\partial_{w_{1}}^{k} \partial_{w^{\prime}}^{\gamma_{0}} f_{0} \prod_{j=1}^{k} \partial^{\gamma_{j}} \beta\right| \leq C^{\prime}|d f| H_{1}^{|\gamma| / 2}
$$

which proves (3.19).

It remains to prove the statements about $\delta_{0}(w)$. Let $G_{1,0}=H_{1} g^{\sharp}=H_{1}(0) g^{\sharp}$ be the signed $G_{1,0}$ distance to $X_{0}$, then it suffices to show that $\delta_{1}(w)=H_{1}^{1 / 2} \delta_{0}(w) \in$ $S\left(1, G_{1,0}\right)$. By chosing

$$
\left\{\begin{array}{l}
z_{1}=H_{1}^{1 / 2}\left(w_{1}-\beta\left(w^{\prime}\right)\right) \\
z^{\prime}=H_{1}^{1 / 2} w^{\prime}
\end{array}\right.
$$

as new coordinates, then we find that $G_{1,0}$ transforms to a uniformly bounded $C^{\infty}$ metric in a neighborhood of the origin. Now $\delta_{1}(z)$ is $\operatorname{sgn}\left(z_{1}\right)$ times the distance to $z_{1}=0$ with respect to this metric, and this is a $C^{\infty}$ function in a sufficiently small neighborhood of the origin. Clearly, $\left|\partial_{z} \delta_{1}\right| \geq c>0$ in a fixed neighborhood of the origin, so Taylor's formula gives $\delta_{1}=\alpha_{0} z_{1}$, where $c / 2 \leq \alpha_{0} \in C^{\infty}$ in that neighborhood. This completes the proof of the proposition. 
We shall compare our metric with the Beals-Fefferman metric $G=H g^{\sharp}$ on $T^{*} \mathbf{R}^{n}$, where

$$
H^{-1}=1+|f|+\left|f^{\prime}\right|^{2} \leq C h^{-1} .
$$

This metric is continuous in $t, \sigma$ temperate on $T^{*} \mathbf{R}^{n}$ and $\sup G / G^{\sigma}=H^{2} \leq 1$. We also have that $f \in S\left(H^{-1}, G\right)$ (see the proof of Lemma 26.10.2 in [10]).

Proposition 3.9. We have that $H^{-1} \leq \mathrm{CH}_{1}^{-1}$ and $M \leq C H_{1}^{-1}$, which implies that $f \in S\left(H_{1}^{-1}, G_{1}\right)$.

Thus, the metric $G_{1}$ gives smaller localization errors than the Beals-Fefferman metric.

Proof. First note that by the Cauchy-Schwarz inequality we have

$$
M=|f|+\left|f^{\prime}\right| H_{1}^{-1 / 2}+\left\|f^{\prime \prime}\right\| H_{1}^{-1}+h^{1 / 2} H_{1}^{-3 / 2} \leq C\left(H^{-1}+H_{1}^{-1}\right) .
$$

Thus we obtain $M \leq C H_{1}^{-1}$ if we show that $H^{-1} \leq C H_{1}^{-1}$. Observe that we only have to prove this when $\left|\delta_{0}\right| \ll H^{-1 / 2}$, since else $H^{-1 / 2} \leq C\left|\delta_{0}\right| \leq C H_{1}^{-1 / 2}$.

If $\left|\delta_{0}\left(w_{0}\right)\right| \leq \kappa H^{-1 / 2}\left(w_{0}\right) \leq C \kappa h^{-1 / 2}$ for $C \kappa<1$, then there exists $w \in f^{-1}(0)$ such that $\left|w-w_{0}\right| \leq \kappa H^{-1 / 2}\left(w_{0}\right)$. For sufficiently small $\kappa$ we find from Taylor's formula and the slow variation that $\left|f\left(w_{0}\right)\right| \leq C \kappa H^{-1}\left(w_{0}\right)$. When $C \kappa \leq 1 / 2$ we obtain that

$$
H^{-1}\left(w_{0}\right) \leq(1-C \kappa)^{-1}\left(1+\left|f^{\prime}\left(w_{0}\right)\right|^{2}\right) \leq 2 H_{1}^{-1}\left(w_{0}\right)
$$

which completes the proof.

\section{The Weight function}

Next, we shall define the weight $m_{\varrho}$ we shall use, for technical reasons it will depend on a parameter $0<\varrho \leq 1$. The weight will essentially measure how much $t \mapsto$ $\delta_{0}(t, w)$ changes between the minima of $t \mapsto H_{1}^{1 / 2}(t, w)$. Since $H_{1}^{1 / 2}$ gives an upper bound on the curvature of the zero set when $H_{1}^{1 / 2} \ll 1$, the weight will give a bound on the sign changes of the symbol (see Lemma 4.4). In the following, we let $\langle s\rangle=1+|s|$.

Definition 4.1. For $0<\varrho \leq 1$ and $\left(t_{0}, w_{0}\right) \in \mathbf{R} \times T^{*} \mathbf{R}^{n}$ we define

$$
m_{ \pm, \varrho}\left(t_{0}, w_{0}\right)=\inf _{ \pm\left(t-t_{0}\right) \geq 0}\left\{\varrho^{2}\left|\delta_{0}\left(t, w_{0}\right)-\delta_{0}\left(t_{0}, w_{0}\right)\right|+H_{1}^{1 / 2}\left(t, w_{0}\right)\left\langle\varrho \delta_{0}\left(t, w_{0}\right)\right\rangle\right\} \leq 1
$$

and

$$
m_{\varrho}=\min \left(\max \left(m_{+, \varrho}, m_{-, \varrho}\right), \varrho^{2}\right) .
$$

thus $m_{1}=\max \left(m_{+, 1}, m_{-, 1}\right)$.

We find that $c h^{1 / 2} \leq m_{ \pm, \varrho} \leq H_{1}^{1 / 2}\left\langle\varrho \delta_{0}\right\rangle$ so

$$
\min \left(c h^{1 / 2}, \varrho^{2}\right) \leq m_{\varrho} \leq \min \left(H_{1}^{1 / 2}\left\langle\varrho \delta_{0}\right\rangle, \varrho^{2}\right) .
$$


by (3.5). Now we have

$$
\begin{aligned}
m_{1}\left(t_{0}, w_{0}\right) \cong \inf _{t^{\prime} \leq t_{0} \leq t^{\prime \prime}}\left\{\delta _ { 0 } \left(t^{\prime \prime},\right.\right. & \left.w_{0}\right)-\delta_{0}\left(t^{\prime}, w_{0}\right) \\
& \left.+H_{1}^{1 / 2}\left(t^{\prime}, w_{0}\right)\left\langle\delta_{0}\left(t^{\prime}, w_{0}\right)\right\rangle+H_{1}^{1 / 2}\left(t^{\prime \prime}, w_{0}\right)\left\langle\delta_{0}\left(t^{\prime \prime}, w_{0}\right)\right\rangle\right\}
\end{aligned}
$$

and thus $m_{1}\left(t_{0}, w_{0}\right) \cong 1$ when $\left|\delta_{0}\left(t, w_{0}\right)\right| \cong H_{1}^{-1 / 2}\left(t, w_{0}\right)$ for $t \geq t_{0}$ or for $t \leq t_{0}$. When $t \mapsto \delta_{0}\left(t, w_{0}\right)$ is constant, we find that $m_{\varrho}$ is proportional to the quasi-convex hull of $t \mapsto H_{1}^{1 / 2}\left(t, w_{0}\right)$ (i.e., it is convex with respect to the constant functions). The weight also has the "convexity property" given by Proposition 4.7: if $\max _{I} m_{1} \gg$ $\min _{I} m_{1}$ on $I=\{(t, w): a \leq t \leq b\}$, then the variation in $t$ of $\delta_{0}$ on $I$ is bounded from below: $\left|\Delta_{I} \delta_{0}\right| \geq c \max _{I} m_{1}>0$. We shall use the parameter $\varrho$ to obtain suitable norms, but this is just a technicality: all $m_{\varrho}$ are equivalent according to the following proposition.

Proposition 4.2. Assume that $\varrho=1$ or $m_{\varrho}\left(t_{0}, w_{0}\right)<\varrho^{2}<1$. For this choice of $\varrho$ there exist $t^{\prime} \leq t_{0} \leq t^{\prime \prime}$ such that

$$
\begin{aligned}
& \left|\delta_{0}\left(t, w_{0}\right)-\delta_{0}\left(t_{0}, w_{0}\right)\right|<\varrho^{-2} m_{\varrho}\left(t_{0}, w_{0}\right) \leq 1 \\
& H_{1}^{1 / 2}\left(t, w_{0}\right)\left\langle\varrho \delta_{0}\left(t, w_{0}\right)\right\rangle<2 m_{\varrho}\left(t_{0}, w_{0}\right) \leq 2 \varrho^{2} .
\end{aligned}
$$

for $t=t^{\prime}$ and $t^{\prime \prime}$. The function $t \mapsto m_{\varrho}(t, w)$ is regulated such that

$$
\varrho_{1}^{2} / \varrho_{2}^{2} \leq m_{\varrho_{1}}(t, w) / m_{\varrho_{2}}(t, w) \leq 1
$$

when $0<\varrho_{1} \leq \varrho_{2} \leq 1$.

We obtain from the proposition that

$$
H_{1}^{1 / 2}\left(t, w_{0}\right)<2 m_{1}\left(t_{0}, w_{0}\right) \text { and }\left|\delta_{0}\left(t, w_{0}\right)\right|<2 m_{1}\left(t_{0}, w_{0}\right) H_{1}^{-1 / 2}\left(t, w_{0}\right)
$$

for $t=t^{\prime}, t^{\prime \prime}$ corresponding to $m_{1}\left(t_{0}, w_{0}\right)$. When $m_{1}\left(t_{0}, w_{0}\right) \ll 1$ we may use Proposition 3.8 at $\left(t^{\prime}, w_{0}\right)$ and $\left(t^{\prime \prime}, w_{0}\right)$. We also obtain from (4.4) that

$$
1 / 2 \leq\left\langle\delta_{0}\left(t, w_{0}\right)\right\rangle /\left\langle\delta_{0}\left(t_{0}, w_{0}\right)\right\rangle \leq 2
$$

for $t=t^{\prime}, t^{\prime \prime}$ corresponding to $m_{1}\left(t_{0}, w_{0}\right)$ in Proposition 4.2, which together with (4.3) gives

$$
H_{1}^{-1 / 2}\left(t_{0}, w_{0}\right) \leq 4 \min \left(H_{1}^{-1 / 2}\left(t^{\prime}, w_{0}\right), H_{1}^{-1 / 2}\left(t^{\prime \prime}, w_{0}\right)\right) .
$$

Proof of Proposition 4.2. We have that $m_{ \pm, \varrho} \leq m_{\varrho}$ when $m_{\varrho}<\varrho^{2}<1$ or when $\varrho=1$. By approximating the limit, we may choose $t^{\prime \prime} \geq t_{0}$ so that

$$
\varrho^{2}\left(\delta_{0}\left(t^{\prime \prime}, w_{0}\right)-\delta_{0}\left(t_{0}, w_{0}\right)\right)+H_{1}^{1 / 2}\left(t^{\prime \prime}, w_{0}\right)\left\langle\varrho \delta_{0}\left(t^{\prime \prime}, w_{0}\right)\right\rangle<m_{+, \varrho}\left(t_{0}, w_{0}\right)+c h^{1 / 2}
$$

where $c$ is chosen as in (3.5). Then we find $\varrho^{2}\left(\delta_{0}\left(t^{\prime \prime}, w_{0}\right)-\delta_{0}\left(t_{0}, w_{0}\right)\right)<m_{+, \varrho}\left(t_{0}, w_{0}\right)$ and $H_{1}^{1 / 2}\left(t^{\prime \prime}, w_{0}\right)\left\langle\varrho \delta_{0}\left(t^{\prime \prime}, w_{0}\right)\right\rangle<m_{+, \varrho}\left(t_{0}, w_{0}\right)+c h^{1 / 2} \leq 2 m_{+, \varrho}\left(t_{0}, w_{0}\right)$, since we have $c h^{1 / 2} \leq m_{+, \varrho}\left(t_{0}, w_{0}\right)$. We similarly obtain these estimates for $m_{-, \varrho}$ with $t^{\prime} \leq t_{0}$, which gives (4.4)-(4.5). 
To prove (4.6) we let $F_{\varrho}(t, s, w)=\varrho^{2}\left|\delta_{0}(s, w)-\delta_{0}(t, w)\right|+H_{1}^{1 / 2}(s, w)\left\langle\varrho \delta_{0}(s, w)\right\rangle$. Then we have $F_{\varrho_{1}} \leq F_{\varrho_{2}}$ and $\varrho_{1}^{2} F_{\varrho_{2}} \leq \varrho_{2}^{2} F_{\varrho_{1}}$ when $\varrho_{1} \leq \varrho_{2}$. Since these estimates are preserved when taking infima and suprema, we obtain (4.6) for $m_{ \pm, \varrho_{j}}$ and $m_{\varrho_{j}}$, $j=1,2$.

To prove that $t \mapsto m_{\varrho}(t, w)$ is a regulated function, it suffices to prove that $t \mapsto m_{ \pm, \varrho}(t, w)$ is a regulated function since this property is preserved when taking maxima and minima. We note that

$$
t \mapsto m_{+, \varrho}\left(t, w_{0}\right)=\inf _{t \leq t^{\prime \prime}}\left\{\varrho^{2} \delta_{0}\left(t^{\prime \prime}, w_{0}\right)+H_{1}^{1 / 2}\left(t^{\prime \prime}, w_{0}\right)\left\langle\varrho \delta_{0}\left(t^{\prime \prime}, w_{0}\right)\right\rangle\right\}-\varrho^{2} \delta_{0}\left(t, w_{0}\right)
$$

and since the infimum is non-decreasing and bounded, we find that this gives a regulated function in $t$. A similar argument works for $m_{-, \varrho}$, which proves the result.

In the following we shall assume the coordinates chosen so that $g^{\sharp}(w)=|w|^{2}$. Observe that $m_{\varrho}$ is not a weight for $G_{1}$, but the following proposition shows that it is a weight for $g_{\varrho}=\varrho^{2} g^{\sharp}$ uniformly in $\varrho$.

Proposition 4.3. We find that there exists $C>0$ such that

$$
m_{\varrho}(t, w) \leq C m_{\varrho}\left(t, w_{0}\right)\left(1+\varrho^{2} g^{\sharp}\left(w-w_{0}\right)\right)
$$

uniformly when $0<\varrho \leq 1$, which implies that $m_{\varrho}$ is a weight for $g_{\varrho}=\varrho^{2} g^{\sharp}$.

Proof. Since $m_{\varrho} \leq \varrho^{2}$ we only have to consider the case when

$$
m_{\varrho}\left(t_{0}, w_{0}\right)<\varrho^{2} .
$$

Now, it suffices to show that

$$
m_{\varrho}\left(t_{0}, w\right) / m_{\varrho}\left(t_{0}, w_{0}\right) \leq C\left(1+\varrho^{2}\left|w-w_{0}\right|^{2}\right) \quad \text { when }\left|w-w_{0}\right| \leq \varrho m_{\varrho}^{-1}\left(t_{0}, w_{0}\right)
$$

uniformly in $0<\varrho \leq 1$. In fact, when $\left|w-w_{0}\right|>\varrho m_{\varrho}^{-1}\left(t_{0}, w_{0}\right)$ we obtain that $\varrho^{2}\left|w-w_{0}\right|^{2}>\varrho^{4} m_{\varrho}^{-2}\left(t_{0}, w_{0}\right)>m_{\varrho}\left(t_{0}, w\right) / m_{\varrho}\left(t_{0}, w_{0}\right)$ by (4.11). Thus (4.10) is trivially satisfied with $C=1$ when $\left|w-w_{0}\right|>\varrho m_{\varrho}^{-1}\left(t_{0}, w_{0}\right)$, thus in the following we shall assume that $\left|w-w_{0}\right| \leq \varrho m_{\varrho}^{-1}\left(t_{0}, w_{0}\right)$.

Now, if (4.10) holds for $m_{\varrho_{0}}$ then it holds for $m_{\varrho}$ when $\varrho_{0} \leq \varrho \leq 1$, with $C$ replaced by $C / \varrho_{0}^{2}$. Thus, in the following we shall assume $0<\varrho \leq \varrho_{0}$ is sufficiently small. Let $m_{\varrho}=m_{\varrho}\left(t_{0}, w_{0}\right)$, then for $\varrho$ small enough one can show that

$$
\left|\delta_{0}\left(t^{\prime}, w\right)-\delta_{0}\left(t^{\prime \prime}, w\right)\right| \leq C_{2}\left(\varrho^{-2} m_{\varrho}+H_{0}^{1 / 2}\left|w^{\prime}\right|^{2}\right) \quad \text { when }\left|w-w_{0}\right| \leq 2 \varrho H_{0}^{-1 / 2},
$$

where $2 \varrho H_{0}^{-1 / 2} \geq \varrho m_{\varrho}^{-1}$ (see the proof of Proposition 6.3 in [7]). We obtain from (4.13) and the monotonicity of $t \mapsto \delta_{0}(t, w)$ that

$$
\varrho^{2}\left|\delta_{0}(t, w)-\delta_{0}\left(t_{0}, w\right)\right| \leq \varrho^{2}\left|\delta_{0}\left(t^{\prime}, w\right)-\delta_{0}\left(t^{\prime \prime}, w\right)\right| \leq C_{2} m_{\varrho}\left(1+\varrho^{2}\left|w^{\prime}\right|^{2}\right)
$$

when $t=t^{\prime}, t^{\prime \prime}$ and $\left|w-w_{0}\right| \leq 2 \varrho H_{0}^{-1 / 2}$. Since $G_{1}$ is slowly varying we find for small $\varrho>0$ that $H_{1}^{1 / 2}(t, w) \leq C_{3} H_{1}^{1 / 2}\left(t, w_{0}\right)$ when $\left|w-w_{0}\right| \leq 2 \varrho H_{0}^{-1 / 2} \leq 2 \varrho H_{1}^{-1 / 2}\left(t, w_{0}\right)$ and $t=t^{\prime}, t^{\prime \prime}$. By the uniform Lipschitz continuity we find

$$
\left\langle\varrho \delta_{0}(t, w)\right\rangle \leq\left\langle\varrho \delta_{0}\left(t, w_{0}\right)\right\rangle\left(1+\varrho\left|w-w_{0}\right|\right) \quad \text { for } t=t^{\prime}, t^{\prime \prime},
$$


which implies that

$$
H_{1}^{1 / 2}(t, w)\left\langle\varrho \delta_{0}(t, w)\right\rangle \leq C_{3} H_{1}^{1 / 2}\left(t, w_{0}\right)\left\langle\varrho \delta_{0}\left(t, w_{0}\right)\right\rangle\left(1+\varrho\left|w-w_{0}\right|\right)
$$

when $t=t^{\prime}, t^{\prime \prime}$ and $\left|w-w_{0}\right| \leq 2 \varrho H_{0}^{-1 / 2}$. By using (4.4)-(4.5), (4.14), (4.16) and taking the infimum we obtain

$m_{ \pm, \varrho}\left(t_{0}, w\right) \leq C_{4} m_{\varrho}\left(t_{0}, w_{0}\right)\left(1+\varrho\left|w-w_{0}\right|\right)^{2}$ when $\left|w-w_{0}\right| \leq \varrho m_{\varrho}^{-1}\left(t_{0}, w_{0}\right) \leq 2 \varrho H_{0}^{-1 / 2}$

uniformly for small $\varrho$. By taking the maximum and then the minimum, we obtain (4.12) and Proposition 4.3.

By using the properties of $m_{\varrho}, H_{1}$ and condition $(\bar{\Psi})$ we can prove the following result (see the proof of Proposition 6.4 in [7]).

Lemma 4.4. There exists $0<\varrho_{0}<1$ and $c_{0}>0$ such that if $m_{1} \leq \varrho_{0}^{2}$ at $\left(t_{0}, w_{0}\right) \in$ $\mathbf{R} \times T^{*} \mathbf{R}^{n}$, then there exist $g^{\sharp}$ orthonormal coordinates so that $w_{0}=\left(z_{1}, 0\right),\left|z_{1}\right|<$ $\left|\delta_{0}\left(t_{0}, w_{0}\right)\right|+1$ and

$$
\operatorname{sgn}\left(w_{1}\right) f\left(t_{0}, w\right) \geq 0 \quad \text { when }\left|w_{1}\right| \geq\left(1+H_{0}^{1 / 2}\left|w^{\prime}\right|^{2}\right) / c_{0} \text { and }|w| \leq c_{0} H_{0}^{-1 / 2}
$$

where $H_{0}^{1 / 2}=\max \left(H_{1}^{1 / 2}\left(t^{\prime}, w_{0}\right), H_{1}^{1 / 2}\left(t^{\prime \prime}, w_{0}\right)\right) \leq 4 m_{1}\left(t_{0}, w_{0}\right) /\left\langle\varrho_{0} \delta_{0}\left(t_{0}, w_{0}\right)\right\rangle \leq 4 \varrho_{0}^{2}$.

In section 5 , we shall choose a fixed $\varrho \ll 1$ in order to get invertible operators and suitable norms. In the following, we shall for simplicity only consider $m_{1}$, since all the $m_{\varrho}$ are equivalent when $\varrho \geq c>0$ by (4.6), this is really no restriction. In order to get lower bounds in terms of the weight $m_{1}$ we need the following proposition, which will be important for the proof.

Proposition 4.5. Let the weight $M$ be given by Definition 3.5. Then there exists $C_{0}>0$ such that

$$
M H_{1}^{3 / 2}\left\langle\delta_{0}\right\rangle \leq C_{0} m_{1}
$$

which gives $S\left(M H_{1}^{3 / 2}, G_{1}\right) \subseteq S\left(m_{1}\left\langle\delta_{0}\right\rangle^{-1}, g^{\sharp}\right)$.

We shall use the following result, for a proof see Proposition 5.2 in [7].

Proposition 4.6. Let $f \in S\left(h^{-1}, h g^{\sharp}\right)$ and let $H_{1}^{1 / 2}$ be given by by Definition 3.4. Assume that

$\operatorname{sgn}\left(w_{1}\right) f(w) \geq 0 \quad$ when $\left(1+H_{0}^{1 / 2}\left|w^{\prime}\right|^{2}\right) / C_{0} \leq\left|w_{1}\right| \leq C_{0} H_{0}^{-1 / 2}$ and $\left|w^{\prime}\right| \leq C_{0} H_{0}^{-1 / 2}$

where $w=\left(w_{1}, w^{\prime}\right)$ and $H_{0}^{1 / 2} \geq h^{1 / 2} / C_{0}$. If $H_{0}^{1 / 2}$ is sufficiently small, then there exist $c_{1}$ and $C_{1}$ such that

$$
\begin{aligned}
& |f(0)| \leq \partial_{w_{1}} f(0) \varrho+C_{1} h^{1 / 2} \varrho^{3} \\
& \left\|f^{\prime \prime}(0)\right\| \leq \partial_{w_{1}} f(0) / \varrho+C_{1} h^{1 / 2} \varrho
\end{aligned}
$$

for any $1 \leq \varrho \leq c_{1} H_{0}^{-1 / 2}$. 
Proof of Proposition 4.5. We shall put $m_{1}=m_{1}\left(t_{0}, w_{0}\right)$, note that if $m_{1} \geq c>0$, then $M H_{1}^{3 / 2}\left\langle\delta_{0}\right\rangle \leq C \leq C m_{1} / c$ at $\left(t_{0}, w_{0}\right)$, since $\left\langle\delta_{0}\right\rangle \leq H_{1}^{-1 / 2}$ and $M \leq C H_{1}^{-1}$ by Proposition 3.9. Thus, we only have to consider the case $m_{1} \ll 1$. Let $0<\varrho_{0}<1$ be given by Lemma 4.4. If $m_{1} \leq \varrho_{0}^{2}$, we may use Lemma 4.4 to obtain $g^{\sharp}$ orthonormal coordinates so that $\left|w_{0}\right| \leq\left|\delta_{0}\left(t_{0}, w_{0}\right)\right|+1 \leq H_{1}^{-1 / 2}\left(t_{0}, w_{0}\right)$ and $f$ satisfies the conditions in Proposition 4.6 with $H_{0}^{1 / 2}=\max \left(H_{1}^{1 / 2}\left(t^{\prime}, w_{0}\right), H_{1}^{1 / 2}\left(t^{\prime \prime}, w_{0}\right)\right) \leq$ $4 m_{\varrho}\left(t_{0}, w_{0}\right) /\left\langle\varrho_{0} \delta_{0}\left(t_{0}, w_{0}\right)\right\rangle \leq 4 \varrho_{0}^{2}$. In the following, we shall omit the dependence on $t_{0}$. Since $\varrho_{0}\left\langle\delta_{0}\right\rangle \leq\left\langle\varrho_{0} \delta_{0}\right\rangle$ we obtain that

$$
H_{0}^{1 / 2}<4 \varrho_{0}^{-1} m_{1} /\left\langle\delta_{0}\left(w_{0}\right)\right\rangle
$$

so we only have to prove the estimate

$$
M H_{1}^{3 / 2} \leq C_{1} H_{0}^{1 / 2} \quad \text { at } w=w_{0}
$$

and we shall start by proving this estimate at $w=0$.

First we observe that if $H_{1}^{1 / 2}(0) \leq C_{0} H_{0}^{1 / 2}$ then $M(0) H_{1}^{3 / 2}(0) \leq C H_{1}^{1 / 2}(0) \leq$ $C C_{0} H_{0}^{1 / 2}$ by Proposition 3.9. Thus, in the following we shall assume $H_{0}^{1 / 2} \leq$ $\varepsilon_{0} H_{1}^{1 / 2}(0)$ for some $\varepsilon_{0}>0$ to be determined later. $>$ From the definition of $M$ we find

$$
M H_{1}^{3 / 2}=|f| H_{1}^{3 / 2}+\left|f^{\prime}\right| H_{1}+\left\|f^{\prime \prime}\right\| H_{1}^{1 / 2}+h^{1 / 2} .
$$

When $\kappa_{0}$ is small enough, we find from Proposition 4.6 that $|f(0)| \leq C\left(\left|f^{\prime}(0)\right|+h^{1 / 2}\right)$ and since $H_{1} \leq 1$ it suffices to estimate $\left\|f^{(k)}(0)\right\|$ for $k=1,2$. We obtain from (3.6) that

$$
\left|f^{\prime}(0)\right| H_{1}(0) \leq 2\left\|f^{\prime \prime}(0)\right\| H_{1}^{1 / 2}(0)+3 h^{1 / 2} .
$$

Thus, we only have to estimate $\left\|f^{\prime \prime}(0)\right\| H_{1}^{1 / 2}(0)$ in order to obtain (4.22) at $w=0$. Now by (4.21) we have

$$
\left\|f^{\prime \prime}(0)\right\| H_{1}^{1 / 2}(0) \leq H_{1}^{1 / 2}(0)\left(\left|f^{\prime}(0)\right| / \varrho+C_{1} h^{1 / 2} \varrho\right)
$$

for any $1 \leq \varrho \leq c_{1} H_{0}^{-1 / 2}$. Thus, if $\varepsilon_{0} \leq c_{1} / 4$, we can choose $\varrho=4 H_{1}^{-1 / 2}(0) \leq$ $4 \varepsilon_{0} H_{0}^{-1 / 2} \leq c_{1} H_{0}^{-1 / 2}$ which gives

$$
\left\|f^{\prime \prime}(0)\right\| H_{1}^{1 / 2}(0) \leq \frac{1}{4}\left|f^{\prime}(0)\right| H_{1}(0)+C h^{1 / 2} \leq \frac{1}{2}\left\|f^{\prime \prime}(0)\right\| H_{1}^{1 / 2}(0)+C_{2} h^{1 / 2} .
$$

by (4.23). This gives $\left\|f^{\prime \prime}(0)\right\| H_{1}^{1 / 2}(0) \leq 2 C_{2} h^{1 / 2} \leq 2 C_{2} C_{0} H_{0}^{1 / 2}$ and (4.22) at $w=0$.

It remains to prove the estimate $M\left(w_{0}\right) H_{1}^{3 / 2}\left(w_{0}\right) \leq C M(0) H_{1}^{3 / 2}(0)$ when $\left|w_{0}\right| \leq$ $H_{1}^{-1 / 2}\left(w_{0}\right)$. By Proposition 3.6 we have that

$$
M\left(w_{0}\right) \leq C M(0)\left(1+H_{1}^{1 / 2}(0)\left|w_{0}\right|\right)^{3}
$$

and

$$
H\left(w_{0}\right) \leq C H(0)\left(1+H_{1}^{1 / 2}\left(w_{0}\right)\left|w_{0}\right|\right)^{2} .
$$


In the case $H_{1}^{1 / 2}(0) \leq H_{1}^{1 / 2}\left(w_{0}\right)$ we find that $\left|w_{0}\right| \leq H_{1}^{-1 / 2}\left(w_{0}\right) \leq H_{1}^{-1 / 2}(0)$ and thus $M\left(w_{0}\right) H_{1}^{3 / 2}\left(w_{0}\right) \leq 64 C^{5 / 2} M(0) H_{1}^{3 / 2}(0)$ by $(4.25)-(4.26)$. When $H_{1}^{1 / 2}\left(w_{0}\right) \leq$ $H_{1}^{1 / 2}(0)$ we don't use (4.26), instead we find from (4.25) that

$$
M\left(w_{0}\right) H_{1}^{3 / 2}\left(w_{0}\right) \leq C M(0) H_{1}^{3 / 2}(0)\left(H_{1}^{1 / 2}\left(w_{0}\right) H_{1}^{-1 / 2}(0)+1\right)^{3} \leq 8 C M(0) H_{1}^{3 / 2}(0)
$$

since $\left|w_{0}\right| \leq H_{1}^{-1 / 2}\left(w_{0}\right)$. This completes the proof of the proposition.

Finally, we shall prove the "convexity property" mentioned earlier.

Proposition 4.7. Let $m_{1}$ be given by Definition 4.1. There exist $\kappa_{0}>1, c_{0}>0$ and $\varepsilon_{0}>0$ such that if $\kappa \geq \kappa_{0}, t^{\prime}<t_{0}<t^{\prime \prime}$ and

$$
m_{1}\left(t_{0}, w_{0}\right)=\kappa \max \left(m_{1}\left(t^{\prime}, w_{0}\right), m_{1}\left(t^{\prime \prime}, w_{0}\right)\right)
$$

then we have

$$
\delta_{0}\left(t^{\prime \prime}, w\right)-\delta_{0}\left(t^{\prime}, w\right) \geq c_{0} m_{1}\left(t_{0}, w_{0}\right)=c_{0} \kappa \max \left(m_{1}\left(t^{\prime}, w_{0}\right), m_{1}\left(t^{\prime \prime}, w_{0}\right)\right)
$$

when $\left|w-w_{0}\right| \leq \varepsilon_{0}$.

Proof. Since $t_{0}<t^{\prime \prime}$ we have by the triangle inequality

$$
\begin{aligned}
m_{+, 1}\left(t_{0}, w_{0}\right) \leq \inf _{t^{\prime \prime} \leq t}\left(\delta_{0}\left(t, w_{0}\right)-\delta_{0}\left(t_{0}, w_{0}\right)\right. & \left.+H_{1}^{1 / 2}(t)\left\langle\delta_{0}\left(t, w_{0}\right)\right\rangle\right) \\
& \leq \delta_{0}\left(t^{\prime \prime}, w_{0}\right)-\delta_{0}\left(t_{0}, w_{0}\right)+m_{+, 1}\left(t^{\prime \prime}, w_{0}\right)
\end{aligned}
$$

and similarly $m_{-, 1}\left(t_{0}, w_{0}\right) \leq \delta_{0}\left(t_{0}, w_{0}\right)-\delta_{0}\left(t^{\prime}, w_{0}\right)+m_{-, 1}\left(t^{\prime}, w_{0}\right)$. Since $m_{ \pm, 1} \leq m_{1}$ we find that

$$
\begin{aligned}
& m_{1}\left(t_{0}, w_{0}\right)=\max \left(m_{-, 1}\left(t_{0}, w_{0}\right), m_{+, 1}\left(t_{0}, w_{0}\right)\right) \\
& \quad \leq \delta_{0}\left(t^{\prime \prime}, w_{0}\right)-\delta_{0}\left(t^{\prime}, w_{0}\right)+\max \left(m_{1}\left(t^{\prime}, w_{0}\right), m_{1}\left(t^{\prime \prime}, w_{0}\right)\right)
\end{aligned}
$$

which gives (4.28) for $w=w_{0}$ with $\kappa_{0}=2$ and $c_{0}=1 / 2$.

If we choose $\varepsilon_{0}>0$ so that $1 / C_{0} \leq m_{1}(t, w) / m_{1}\left(t, w_{0}\right) \leq C_{0}$ for $\left|w-w_{0}\right| \leq \varepsilon_{0}$ and all $t$, then we obtain (4.28) with $\kappa_{0}=2 C_{0}^{2}$ and $c_{0}=\left(2 C_{0}\right)^{-1}$.

\section{The Pseudo-Sign}

In order to construct a pseudo-sign we shall use the Wick quantization. For $a(x, \xi) \in$ $L^{\infty}\left(T^{*} \mathbf{R}^{n}\right)$ we define the Wick quantization:

$$
a^{W i c k}\left(x, D_{x}\right) u(x)=\int_{T^{*} \mathbf{R}^{n}} a(y, \eta) \Sigma_{y, \eta}^{w}\left(x, D_{x}\right) u(y) d y d \eta \quad u \in \mathcal{S}\left(\mathbf{R}^{n}\right)
$$

using the projections $\Sigma_{y, \eta}^{w}\left(x, D_{x}\right)$ with symbol

$$
\Sigma_{y, \eta}(x, \xi)=\pi^{-n} \exp \left(-g^{\sharp}(x-y, \xi-\eta)\right)=\pi^{-n} \exp \left(-|x-y|^{2}-|\xi-\eta|^{2}\right) .
$$


We find that $a^{\text {Wick }}: \mathcal{S}\left(\mathbf{R}^{n}\right) \mapsto \mathcal{S}^{\prime}\left(\mathbf{R}^{n}\right)$ is symmetric on $\mathcal{S}\left(\mathbf{R}^{n}\right)$ if $a$ is real valued,

$$
a \geq 0 \quad \text { in } L^{\infty}\left(T^{*} \mathbf{R}^{n}\right) \Rightarrow\left\langle a^{\text {Wick }}\left(x, D_{x}\right) u, u\right\rangle \geq 0 \quad \text { for } u \in \mathcal{S}\left(\mathbf{R}^{n}\right)
$$

(see [15, Proposition 4.2]). We obtain from the definition that $a^{W i c k}=a_{0}^{w}$ where

$$
a_{0}(w)=\pi^{-n} \int a(z) \exp \left(-|w-z|^{2}\right) d z
$$

is the Gaussian regularization. Observe that real Wick symbols have real Weyl symbols.

Proposition 5.1. Assume that $a \in L^{\infty}\left(T^{*} \mathbf{R}^{n}\right)$, then $a_{0}^{w}=a^{\text {Wick }}$ where $a_{0}$ is given by (5.2). If $|a| \leq C M$ then we find that $a_{0} \in S\left(M, g^{\sharp}\right)$. If also $a \in S\left(M, G_{1}\right)$ in $a$ $G_{1}$ ball of fixed radius with center $w$, then $a_{0} \cong$ a modulo symbols in $S\left(H_{1} M, G_{1}\right)$ in a fixed $G_{1}$ neighborhood of $w$. If $a \geq M$ we obtain $a_{0} \geq c M$, and if $a \geq M$ in $a G_{1}$ ball of fixed radius with center $w$ then $a_{0} \geq c M-C H_{1} M$ in a fixed $G_{1}$ neighborhood of $w$, for some constants $c, C>0$. If $|d a| \leq C$ almost everywhere, then $a_{0} \in S^{+}\left(1, g^{\sharp}\right)$.

Proof. Since $a$ is measurable satisfying $|a| \leq C M$, we find that $a^{\text {Wick }}=a_{0}^{w}$ where $a_{0}$ is given by (5.2). Since $M(z) \leq C M(w)(1+|z-w|)^{3}$ by (3.13), we obtain that $a_{0}(w)=\mathcal{O}(M(w))$. By differentiating on the exponential factor, we find $a_{0} \in$ $S\left(M, g^{\sharp}\right)$, and similarly we find that $a_{0} \geq M / C$ if $a \geq M$.

If $a \in S\left(M, H_{1} g^{\sharp}\right)$ in a $G_{1}$ ball of radius $c>0$ and center at $w$, then we write

$$
\begin{array}{rl}
a_{0}(w)=\pi^{-n} \int_{T^{*} \mathbf{R}^{n}} & a(z) \exp \left(-|w-z|^{2}\right) d z \\
=\pi^{-n} \int_{|w-z| \leq c H_{1}^{-1 / 2}(w) / 2} a(z) \exp \left(-|w-z|^{2}\right) d z & \\
& +\pi^{-n} \int_{|w-z| \geq c H_{1}^{-1 / 2}(w) / 2} a(z) \exp \left(-|w-z|^{2}\right) d z
\end{array}
$$

where the last term is $\mathcal{O}\left(H_{1}^{N}(w) M(w)\right)$ for any $N$. Thus, after multiplying with a cut-off function, we may assume that $a \in S\left(M, G_{1}\right)$ everywhere. Taylor's formula gives

$$
\begin{aligned}
a_{0}(w)=\pi^{-n} \int_{T^{*} \mathbf{R}^{n}} a(w & +z) \exp \left(-|z|^{2}\right) d z \\
= & a(w)+\pi^{-n} \int_{0}^{1} \int_{T^{*} \mathbf{R}^{n}}(1-\theta)\left\langle a^{\prime \prime}(w+\theta z) z, z\right\rangle e^{-|z|^{2}} d z d \theta
\end{aligned}
$$

where $a^{\prime \prime} \in S\left(M H_{1}, G_{1}\right)$ since $G_{1}=H_{1} g^{\sharp}$. Since differentiation commutes with convolution, we find from (3.12)-(3.13) that $a_{0}(w) \cong a(w)$ modulo symbols in $S\left(H_{1} M, G_{1}\right)$. Similarly, we obtain that $a_{0} \geq c M$ modulo $S\left(H_{1} M, g^{\sharp}\right)$ for some $c>0$ if $a \geq M$ in a fixed $G_{1}$ ball. Since $d a_{0}(w)=\pi^{-n} \int_{T^{*} \mathbf{R}^{n}} d a(z) \exp \left(-|w-z|^{2}\right) d z$, we obtain the last statement. 
Remark 5.2. If $a(t, w)$ and $g(t, w) \in L^{\infty}\left(\mathbf{R} \times T^{*} \mathbf{R}^{n}\right)$ such that $\partial_{t} a(t, w) \geq g(t, w)$ in $\mathcal{D}^{\prime}(\mathbf{R})$ for almost all $w \in T^{*} \mathbf{R}^{n}$, then we find $\left\langle\partial_{t}\left(a^{\text {Wick }}\right) u, u\right\rangle \geq\left\langle g^{\text {Wick }} u, u\right\rangle$ in $\mathcal{D}^{\prime}(\mathbf{R})$ when $u \in \mathcal{S}\left(\mathbf{R}^{n}\right)$.

In fact, the condition means that

$$
-\int a(t, w) \phi^{\prime}(t) d t \geq \int g(t, w) \phi(t) d t \quad 0 \leq \phi \in C_{0}^{\infty}(\mathbf{R})
$$

for almost all $w \in T^{*} \mathbf{R}^{n}$, and then (5.1) gives

$-\int\left\langle a^{W i c k}\left(t, x, D_{x}\right) u, u\right\rangle \phi^{\prime}(t) d t \geq \int\left\langle g^{W i c k}\left(t, x, D_{x}\right) u, u\right\rangle \phi(t) d t \quad 0 \leq \phi \in C_{0}^{\infty}(\mathbf{R})$ for $u \in \mathcal{S}\left(\mathbf{R}^{n}\right)$.

We are going to use the symbol classes $S\left(m_{\varrho}^{k}, g_{\varrho}\right)$ where $g_{\varrho}=\varrho^{2} g^{\sharp}$ and $m_{\varrho}$ is given by Definition 4.1. Observe that $S\left(m_{\varrho}^{k}, g_{\varrho}\right)=S\left(m_{1}^{k}, g^{\sharp}\right)$ for all $0<\varrho \leq 1$. In fact, $g_{\varrho}=\varrho^{2} g^{\sharp}$ and $m_{\varrho} \leq m_{1} \leq \varrho^{-2} m_{\varrho}$ by (4.6). By [2, Corollary 6.7] we can define Sobolev spaces $H\left(m_{\varrho}^{k}, g_{\varrho}\right)$ with the following properties: $\mathcal{S}$ is dense in $H\left(m_{\varrho}^{k}, g_{\varrho}\right)$, the dual of $H\left(m_{\varrho}^{k}, g_{\varrho}\right)$ is naturally identified with $H\left(m_{\varrho}^{-k}, g_{\varrho}\right)$, and

$$
u \in H\left(m_{\varrho}^{k}, g_{\varrho}\right) \Longleftrightarrow a^{w} u \in L^{2}=H\left(1, g_{\varrho}\right) \quad \forall a \in S\left(m_{\varrho}^{k}, g_{\varrho}\right)
$$

and then $u=a_{0}^{w} v$ for some $a_{0} \in S\left(m_{\varrho}^{-k}, g_{\varrho}\right)$ and $v \in L^{2}$. Observe that $H\left(m_{\varrho}^{k}, g_{\varrho}\right)=$ $H\left(m_{1}^{k}, g^{\sharp}\right)$ for all $0<\varrho \leq 1$, but not uniformly. We also find from [2, Corollary 4.4] that $a^{w}$ is bounded as an operator:

$$
u \in H\left(m_{\varrho}^{j}, g_{\varrho}\right) \mapsto a^{w} u \in H\left(m_{\varrho}^{j-k}, g_{\varrho}\right) \quad \text { when } a \in S\left(m_{\varrho}^{k}, g_{\varrho}\right),
$$

and the bound only depends on the seminorms of $a$ in $S\left(m_{\varrho}^{k}, g_{\varrho}\right)$.

Let $\mu_{\varrho}^{w}=m_{\varrho}^{\text {Wick }}$, i.e.,

$$
\mu_{\varrho}(t, w)=\pi^{-n} \int_{T^{*} \mathbf{R}^{n}} m_{\varrho}(t, z) \exp \left(-|w-z|^{2}\right) d z .
$$

Since $m_{\varrho}$ satisfies (4.10) we find from Proposition 5.1 that

$$
m_{\varrho} / c_{0} \leq \mu_{\varrho} \in L^{\infty}\left(\mathbf{R}, S\left(m_{\varrho}, g_{\varrho}\right)\right)
$$

uniformly for $0<\varrho \leq 1$ for some $c_{0}>0$.

Proposition 5.3. Assume that the symbol $\mu=\mu_{\varrho_{0}} \in L^{\infty}\left(\mathbf{R}, S\left(m_{1}, g^{\sharp}\right)\right)$ is given by (5.5) with $\varrho=\varrho_{0} \ll 1$, thus $\mu^{w}=m_{\varrho_{0}}^{\text {Wick }} \leq m_{1}^{\text {Wick }}$. Then there exist positive constants $c_{0}, c_{1}$ and $C_{0}$ such that

$$
c_{0} h^{1 / 2}\|u\|^{2} \leq c_{1}\|u\|_{H\left(m_{1}^{1 / 2}\right)}^{2} \leq\left\langle\mu^{w} u, u\right\rangle \leq C_{0}\|u\|_{H\left(m_{1}^{1 / 2}\right)}^{2} .
$$

The proof relies on the fact that when $0<\varrho \ll 1$ we have

$$
\begin{aligned}
& \left(m_{\varrho}^{1 / 2}\right)^{w}\left(m_{\varrho}^{-1 / 2}\right)^{w}=\gamma_{\varrho}^{w} \text { is uniformly invertible in } L^{2} \\
& \frac{1}{2} \leq\left(m_{\varrho}^{-1 / 2}\right)^{w} m_{\varrho}^{w}\left(m_{\varrho}^{-1 / 2}\right)^{w} \leq 2 \quad \text { in } L^{2}
\end{aligned}
$$

when $|t| \leq 1$ (see the proof of [7, Proposition 7.4]).

Next, we shall construct a perturbation $B(t, w)=\delta_{0}(t, w)+\varrho_{0}(t, w)$ of $\delta_{0}$ so that $b^{w}=B^{\text {Wick }}$ could be used as the pseudo-sign in Proposition 2.9. 
Proposition 5.4. Assume that $\delta_{0}$ is given by Definition 3.2 and $m_{1}$ is given by Definition 4.1. Then there exist a positive constant $C_{1}$ and a real valued $\varrho_{0}(t, w) \in$ $L^{\infty}\left(\mathbf{R} \times T^{*} \mathbf{R}^{n}\right)$ such that

$$
\begin{aligned}
& \left|\varrho_{0}\right| \leq C_{1} m_{1} \\
& \partial_{t}\left(\delta_{0}+\varrho_{0}\right) \geq m_{1} / C_{1}
\end{aligned}
$$

in $\mathcal{D}^{\prime}(\mathbf{R})$ when $|t|<1$. We also have that $t \mapsto \varrho_{0}(t, w)$ is a regulated function, $\forall w \in T^{*} \mathbf{R}^{n}$, and $w \mapsto \varrho_{0}(t, w) \in \operatorname{Lip}\left(T^{*} \mathbf{R}^{n}\right)$ uniformly for almost all $|t| \leq 1$.

The proof is long and technical (see the proof of [7, Proposition 8.1]), but the idea is as follows. When $t \mapsto m_{1}(t, w)$ has a approximate minimum at $t=t_{0}$ in the sense that $m(s) \leq C m(t)$ when $t \leq s \leq t_{0}$ or $t_{0} \leq s \leq t$, we may take $\varrho_{0}(t, w)=c \int_{t_{0}}^{t} m_{1}(s, w) d s$ since $t \mapsto \delta_{0}(t, w)$ is non-decreasing. In general, we have to split the interval $[-1,1]$ into subintervals where $t \mapsto m_{1}(t, w)$ has approximate maximum and minimum, and use the "convexity property" of $t \mapsto \delta_{0}(t, w)$ given by Proposition 4.7 in order to interpolate $\delta_{0}$ at the approximate maxima of $t \mapsto \delta_{0}(t, w)$.

By Proposition 5.4 and Remark 5.2 we obtain lower bounds on $\partial_{t} B^{\text {Wick }}$ if $B=$ $\delta_{0}+\varrho_{0}$. But in order to prove Proposition 2.9 we also have to obtain lower bounds on $\operatorname{Re} B^{W i c k} f^{w}$. To obtain that, we have to compute the Weyl symbol for the pseudo-sign $B^{\text {Wick }}$.

Proposition 5.5. Let $B=\delta_{0}+\varrho_{0}$, where $\delta_{0}$ is given by Definition 3.2 and $\varrho_{0}(t, w)$ is the real valued symbol given by Proposition 5.4, satisfying $\left|\varrho_{0}(t, w)\right| \leq C m_{1}(t, w)$ for almost all $|t| \leq 1$. Then we find

$$
B^{W i c k}=b^{w} \quad|t| \leq 1
$$

where $b=\delta_{1}+\varrho_{1} \in S\left(H_{1}^{-1 / 2}, g^{\sharp}\right) \bigcap S^{+}\left(1, g^{\sharp}\right)$ is real valued and regulated in $t$, and $\varrho_{1} \in S\left(m_{1}, g^{\sharp}\right) \subseteq S\left(H_{1}^{1 / 2}\left\langle\delta_{0}\right\rangle, g^{\sharp}\right)$ for almost all $|t| \leq 1$. There also exists a positive constant $\kappa_{2}$ with the following properties. For any $\lambda>0$, there exists $c_{\lambda}>0$ such that if $\left|\delta_{0}\right| \geq \lambda H_{1}^{-1 / 2}$ and $H_{1}^{1 / 2} \leq c_{\lambda}$ then $|b| \geq \kappa_{2} \lambda H_{1}^{-1 / 2}$. If $H_{1}^{1 / 2}\left(t, w_{0}\right) \leq \kappa_{2}$ and $\left|\delta_{0}\left(t, w_{0}\right)\right| \leq \kappa_{2} H_{1}^{-1 / 2}\left(t, w_{0}\right)$ then we have $S\left(H_{1}^{-1 / 2}, G_{1}\right) \ni \delta_{1}(t, w)=\delta_{0}(t, w)+$ $\varrho_{2}(t, w)$ when $\left|w-w_{0}\right| \leq \kappa_{2} H_{1}^{-1 / 2}\left(t, w_{0}\right)$ with real valued $\varrho_{2}(t, w) \in S\left(H_{1}^{1 / 2}, G_{1}\right)$.

Proof. Let $\delta_{0}^{\text {Wick }}=\delta_{1}^{w}$ and $\varrho_{0}^{\text {Wick }}=\varrho_{1}^{w}$. Since $\left|\delta_{0}\right| \leq C H_{1}^{-1 / 2},\left|\varrho_{0}\right| \leq C m_{1}$ and the symbols are real valued, we obtain from Proposition 5.1 and (4.3) that $\delta_{1} \in$ $S\left(H_{1}^{-1 / 2}, g^{\sharp}\right)$ and $\varrho_{1} \in S\left(m_{1}, g^{\sharp}\right) \subseteq S\left(H_{1}^{1 / 2}\left\langle\delta_{0}\right\rangle, g^{\sharp}\right)$ are real valued for almost all $|t| \leq 1$. Observe that $m_{1} \leq 1$, and since $\left|\delta_{0}^{\prime}\right| \leq 1$ almost everywhere we find that $b \in S^{+}\left(1, g^{\sharp}\right)$ for almost all $|t| \leq 1$ by Proposition 5.1. Since $\delta_{0}(t, w)$ and $\varrho_{0}(t, w)$ are regulated in $t$, we find from (5.2) that the same holds for $\delta_{1}(t, w)$ and $\varrho_{1}(t, w)$.

When $\left|\delta_{0}\right| \geq \lambda H_{1}^{-1 / 2}$ at $(t, w), \lambda>0$, then by the Lipschitz continuity and slow variation we find that $\left|\delta_{0}\right| \geq \lambda H_{1}^{-1 / 2} / C_{0}$ in a $G_{1}$ neighborhood $\omega$ of $(t, w)$ (depending on $\lambda$ ). Since $\left|\varrho_{0}\right| \leq C H_{1}^{1 / 2}\left\langle\delta_{0}\right\rangle$ we find by the slow variation that $\mid \delta_{0}+$ $\varrho_{0} \mid \geq \lambda H_{1}^{-1 / 2} / 2 C_{0}$ in $\omega$ when $H_{1}^{1 / 2}(t, w)$ is small enough. Proposition 5.1 gives $|b| \geq c \lambda H_{1}^{-1 / 2} / 2 C_{0}-C \lambda H_{1}^{1 / 2} / 2 C_{0} \geq c \lambda H_{1}^{-1 / 2} / 3 C_{0}$ at $(t, w)$ when $H_{1}^{1 / 2}(t, w)$ is small enough. 
If $\left|\delta_{0}\right| \leq \kappa_{2} H_{1}^{-1 / 2}$ and $H_{1}^{1 / 2} \leq \kappa_{2}$ for sufficiently small $\kappa_{2}>0$, then $\left|\delta_{0}\right| \leq$ $C_{0} \kappa_{2} H_{1}^{-1 / 2}$ and $H_{1}^{1 / 2} \leq C_{0} \kappa_{2}$ in a fixed $G_{1}$ neighborhood. Thus, for $\kappa_{2} \ll 1$ we obtain that $\delta_{0} \in S\left(H_{1}^{-1 / 2}, G_{1}\right)$ in a fixed $G_{1}$ neighborhood. Then we obtain the last statement from Proposition 5.1, which completes the proof.

Next, we shall obtain lower bounds on $\operatorname{Re} B^{W i c k} f^{w}=\operatorname{Re} b^{w} f^{w}$, and finally prove Proposition 2.9.

Proposition 5.6. Assume that $b=\delta_{1}+\varrho_{1}$ is given by Proposition 5.5. Then we have

$$
\operatorname{Re}\left\langle\left.\left(b^{w} f^{w}\right)\right|_{t} u, u\right\rangle \geq\left\langle C_{t}^{w} u, u\right\rangle \quad \forall u \in C_{0}^{\infty}\left(\mathbf{R}^{n}\right) \quad \text { for almost all }|t| \leq 1
$$

where $C_{t} \in S\left(m_{1}(t), g^{\sharp}\right)$ has uniformly bounded seminorms.

The proposition is proved by localizing with respect to the metric $G_{1}$ for fixed $t$. Observe that we may ignore terms in Op $S\left(M H_{1}^{3 / 2}\left\langle\delta_{0}\right\rangle, g^{\sharp}\right) \subseteq \mathrm{Op} S\left(m_{1}, g^{\sharp}\right)$ by Proposition 4.5 , which makes the localization possible. Also, when $H_{1} \cong 1$ we have bf $\in S\left(M H_{1}^{N}, g^{\sharp}\right)$ for any $N$, thus we may assume $H_{1} \ll 1$.

By the slow variation of $G_{1}$ and the uniform Lipschitz continuity of $w \mapsto \delta_{0}(w)$, we may consider the domains where $\left|\delta_{0}\right| \gtrless \kappa H_{1}^{-1 / 2}$ for $\kappa \ll 1$. When $\left|\delta_{0}\right| \geq \kappa H_{1}^{-1 / 2}$ and $H_{1} \ll 1$ we use that $b f$ is a product of a non-negative symbol and an elliptic symbol. Moreover, we find that $M H_{1}^{3 / 2}\left\langle\delta_{0}\right\rangle \cong M H_{1}$ in this case, so by perturbing the Fefferman-Phong estimate for $f^{w}$ we obtain the lower bounds in this case.

When $\left|\delta_{0}\right| \ll H_{1}^{-1 / 2}$ and $H_{1} \ll 1$ we find that $\delta_{0} \in S\left(H_{1}^{-1 / 2}, G_{1}\right)$ but bf $¥ 0$. By completing the square and taking an approximate square root, we obtain the lower bounds by using the calculus (see the proof of Theorem 9.1 in [7]).

Proof of Proposition 2.9. Let $B^{\text {Wick }}=\left(\delta_{0}+\varrho_{0}\right)^{\text {Wick }}$ be the pseudo-sign, where $\delta_{0}+$ $\varrho_{0}$ is given by Proposition 5.4. We find that $B^{W i c k}=b^{w}=\left(\delta_{1}+\varrho_{1}\right)^{w}$ where $b(t, w) \in L^{\infty}\left(\mathbf{R}, S\left(H_{1}^{-1 / 2}, g^{\sharp}\right)+S^{+}\left(1, g^{\sharp}\right)\right)$ is given by Proposition 5.5 for $|t| \leq 1$. Now $\partial_{t}\left(\delta_{0}+\varrho_{0}\right) \geq m_{1} / C_{1}$ in $\mathcal{D}^{\prime}(\mathbf{R})$ when $|t|<1$ by Proposition 5.4. Let $\mu^{w} \in$ $L^{\infty}\left(\mathbf{R}\right.$, Op $\left.S\left(m_{1}, g^{\sharp}\right)\right)$ be given by Proposition 5.3 , then $m_{1}^{\text {Wick }} \geq \mu^{w}$. Thus we find by Remark 5.2 that

$$
\partial_{t}\left\langle b^{w} u, u\right\rangle=\left\langle\partial_{t} B^{W i c k} u, u\right\rangle \geq C_{1}^{-1}\left\langle\mu^{w} u, u\right\rangle \quad \text { in } \mathcal{D}^{\prime}(\mathbf{R})
$$

when $u \in C_{0}^{\infty}\left(\mathbf{R}^{n}\right)$. We obtain from Proposition 5.3 that there exist positive constants $c_{0}$ and $c_{1}$ so that

$$
\left\langle\mu^{w} u, u\right\rangle \geq c_{1}\|u\|_{H\left(m_{1}^{1 / 2}\right)}^{2} \geq c_{0} h^{1 / 2}\|u\|^{2} \quad u \in C_{0}^{\infty}\left(\mathbf{R}^{n}\right) .
$$

Here $\|u\|_{H\left(m_{1}^{1 / 2}\right)}$ is the norm of the Sobolev space $H\left(m_{1}^{1 / 2}, g^{\sharp}\right)=H\left(m_{1}^{1 / 2}\right)$ given by (5.3) with $\varrho=1$ and $k=1 / 2$. By Proposition 5.6 we find for almost all $|t| \leq 1$ that

$$
\operatorname{Re}\left\langle\left.\left(B^{W i c k} f^{w}\right)\right|_{t} u, u\right\rangle=\operatorname{Re}\left\langle\left.\left(b^{w} f^{w}\right)\right|_{t} u, u\right\rangle \geq\left\langle C_{t}^{w} u, u\right\rangle \quad u \in C_{0}^{\infty}\left(\mathbf{R}^{n}\right)
$$


with $C_{t} \in S\left(m_{1}(t), g^{\sharp}\right)$ uniformly. Thus we obtain from (5.4) and duality that there exists a positive constant $c_{2}$ such that

$$
\left|\left\langle C_{t}^{w} u, u\right\rangle\right| \leq\|u\|_{H\left(m_{1}^{1 / 2}\right)}\left\|C_{t}^{w} u\right\|_{H\left(m_{1}^{-1 / 2}\right)} \leq c_{2}\|u\|_{H\left(m_{1}^{1 / 2}\right)}^{2} \leq c_{2}\left\langle\mu^{w} u, u\right\rangle / c_{1}
$$

for $u \in C_{0}^{\infty}\left(\mathbf{R}^{n}\right)$ and almost all $|t| \leq 1$. We obtain Proposition 2.9 from (5.12)(5.15), which completes the proof of Theorem 1.1 and the Nirenberg-Treves conjecture.

\section{References}

[1] Richard Beals and Charles Fefferman, On local solvability of linear partial differential equations, Ann. of Math. 97 (1973), 482-498.

[2] Jean-Michel Bony and Jean-Yves Chemin, Espace fonctionnels associés au calcul de Weyl-Hörmander, Bull. Soc. Math. France 122 (1994), 77-118.

[3] Nils Dencker, On the propagation of singularities for pseudo-differential operators of principal type, Ark. Mat. 20 (1982), 23-60.

[4] The solvability of non $L^{2}$ solvable operators, Journees "Équations aux Dérivées Partielles", St. Jean de Monts, France, 1996.

[5] _ A sufficient condition for solvability, International Mathematics Research Notices 1999:12 (1999), 627-659.

[6] _ On the sufficiency of condition ( $\Psi)$, Report 2001:11, Centre for Mathematical Sciences, Lund University.

[7] Mittag-Leffler, 2002/2003 fall.

[8] Jean Dieudonné, Foundations of modern analysis, Academic Press, New York and London, 1960.

[9] Lars Hörmander, The Weyl calculus of pseudo-differential operators, Comm. Partial Differential Equations 32 (1979), 359-443.

[10] , The analysis of linear partial differential operators, vol. I-IV, Springer Verlag, Berlin, 1983-1985.

[11]_ Notions of convexity, Birkhäuser, Boston, 1994.

[12] _ On the solvability of pseudodifferential equations, Structure of solutions of differential equations (M. Morimoto and T. Kawai, eds.), World Scientific, New Jersey, 1996, pp. 183-213.

[13] Nicolas Lerner, Sufficiency of condition $(\Psi)$ for local solvability in two dimensions, Ann. of Math. 128 (1988), 243-258. 
[14] Nonsolvability in $L^{2}$ for a first order operator satisfying condition $(\Psi)$, Ann. of Math. 139 (1994), 363-393.

[15] _ Energy methods via coherent states and advanced pseudo-differential calculus, Multidimensional complex analysis and partial differential equations (P. D. Cordaro, H. Jacobowitz, and S. Gidikin, eds.), Amer. Math. Soc., Providence, R.I., USA, 1997, pp. 177-201.

[16] _ Perturbation and energy estimates, Ann. Sci. École Norm. Sup. 31 (1998), 843-886.

[17] _ Factorization and solvability, Preprint.

[18]_, Private communication.

[19] Louis Nirenberg and François Treves, On local solvability of linear partial differential equations. Part I: Necessary conditions, Comm. Partial Differential Equations 23 (1970), 1-38, Part II: Sufficient conditions, Comm. Pure Appl. Math. 23 (1970), 459-509; Correction, Comm. Pure Appl. Math. 24 (1971), $279-288$.

[20] Jean-Marie Trépreau, Sur la résolubilité analytique microlocale des opérateurs pseudodifférentiels de type principal, Ph.D. thesis, Université de Reims, 1984.

Centre of Mathematical Sciences, University of Lund, Box 118, S-221 00 Lund, Sweden

dencker@maths. 1th.se 